Research Article

\title{
Permanence and Almost Periodic Solutions of a Discrete Ratio-Dependent Leslie System with Time Delays and Feedback Controls
}

\author{
Gang Yu and Hongying Lu \\ School of Mathematics and Quantitative Economics, Dongbei University of Finance and Economics, \\ Dalian 116025, China \\ Correspondence should be addressed to Hongying Lu, hongyinglu543@163.com
}

Received 10 July 2012; Accepted 2 September 2012

Academic Editor: Wolfgang Ruess

Copyright (C) 2012 G. Yu and H. Lu. This is an open access article distributed under the Creative Commons Attribution License, which permits unrestricted use, distribution, and reproduction in any medium, provided the original work is properly cited.

We consider a discrete almost periodic ratio-dependent Leslie system with time delays and feedback controls. Sufficient conditions are obtained for the permanence and global attractivity of the system. Furthermore, by using an almost periodic functional Hull theory, we show that the almost periodic system has a unique globally attractive positive almost periodic solution.

\section{Introduction}

Among the relationships between the species living in the same outer environment, the predator-prey theory plays an important and fundamental role. The predator-prey models have been extensively studied by many scholars [1-4]. Under the assumption that reduction in a predator population has a reciprocal relationship with per capita availability of its preferred food, Leslie [5] proposed the famous Leslie-Gower predator-prey model where the carry capacity of the predator's environment is proportional to the number of prey:

$$
\begin{gathered}
\dot{x}_{1}=x_{1}\left[b-a x_{1}\right]-c x_{1} x_{2}, \\
\dot{x}_{2}=x_{2}\left[g-f \frac{x_{2}}{x_{1}}\right],
\end{gathered}
$$

where $x_{1}$ and $x_{2}$ represent prey and predator densities at time $t$, respectively. $c x_{1}$ is the predator's rate of feeding upon prey, that is, the so-called predator's functional response. 
If we assume that the predator consumes the prey according to the functional response $p\left(x_{1}\right)$, then system (1.1) formulates as the following:

$$
\begin{gathered}
\dot{x}_{1}=x_{1}\left[b-a x_{1}\right]-p\left(x_{1}\right) x_{2}, \\
\dot{x}_{2}=x_{2}\left[g-f \frac{x_{2}}{x_{1}}\right],
\end{gathered}
$$

where $p\left(x_{1}\right)$ is prey-dependent functional responses. Owing to its theoretical and practical significance, system (1.2) and its various generalized forms have been studied extensively and seen great progress (see, for example, [6-9]).

However, in the study of the dynamic behaviors of predator-prey system, many scholars argued that the ratio-dependent predator-prey systems are more realistic $[10,11]$. A ratio-dependent predator-prey system with Leslie-Gower term takes the form of

$$
\begin{gathered}
\dot{x}_{1}=x_{1}\left[b-a x_{1}\right]-p\left(\frac{x_{1}}{x_{2}}\right) x_{2}, \\
\dot{x}_{2}=x_{2}\left[g-f \frac{x_{2}}{x_{1}}\right] .
\end{gathered}
$$

Though much progress has been seen in the asymptotic convergence of solutions of population systems, such systems are not well studied in the sense that most results are continuous-time cases related. Already, many scholars have paid attention to the nonautonomous discrete population models, since the discrete time models governed by difference equation are more appropriate than the continuous ones when the populations have a short life expectancy, nonoverlapping generations in the real world (see [12-23]). Furthermore, the asymptotic convergence of solutions of difference equations with delay is one of the most important topics in the study of population dynamics, and many excellent results have already been obtained and seen great progress (see [24-26] and the references cited therein).

Since time delays occur so often in nature, a number of ecological systems can be described as systems with time delays (see [14, 15, 19, 21-27]). One of the most important problems for this type of system is to analyze the effect of time delays on the stability of the system. Furthermore, as we know, ecological systems in the real world are often distributed by unpredictable forces which can result in changes in biological parameters such as survival rates, so it is reasonable to study models with control variables which are so-called disturbance functions $[22,23]$.

So it is very interesting to study dynamics of the following discrete ratio-dependent Leslie system with time delays and feedback controls:

$$
\begin{gathered}
x_{1}(k+1)=x_{1}(k) \exp \left[b(k)-a(k) x_{1}\left(k-\tau_{1}\right)-\frac{c(k) x_{1}(k) x_{2}(k)}{h^{2} x_{2}^{2}(k)+x_{1}^{2}(k)}-d(k) u_{1}\left(k-\sigma_{1}\right)\right], \\
x_{2}(k+1)=x_{2}(k) \exp \left[g(k)-f(k) \frac{x_{2}\left(k-\tau_{2}\right)}{x_{1}\left(k-\tau_{2}\right)}-p(k) u_{2}\left(k-\sigma_{2}\right)\right], \\
\Delta u_{i}(k)=-\alpha_{i}(k) u_{i}(k)+\beta_{i}(k) x_{i}\left(k-\rho_{i}\right), \quad i=1,2,
\end{gathered}
$$


where $x_{i}(k), i=1,2$ stand for the density of the prey and the predator at time $k$, respectively. $u_{i}(k), i=1,2$ are the control variables at time $k . h^{2}$ is a positive constant, denoting the constant of capturing half-saturation.

In this paper, we are concerned with the effects of the almost periodicity of ecological and environmental parameters and time delays on the global dynamics of the discrete ratiodependent Leslie systems with feedback controls. To do so, for system (1.4) we always assume that for $i=1,2, k \in Z$

$\left(H_{1}\right) a(k), b(k), c(k), d(k), g(k), f(k), p(k), \alpha_{i}(k), \beta_{i}(k)$ are all bounded nonnegative almost periodic sequences such that

$$
\begin{aligned}
& 0<a^{l} \leq a^{u}, \quad 0<b^{l} \leq b^{u}, \quad 0<c^{l} \leq c^{u}, \quad 0<d^{l} \leq d^{u}, \quad 0<g^{l} \leq g^{u}, \\
& 0<f^{l} \leq f^{u}, \quad 0<p^{l} \leq p^{u}, \quad 0<\alpha_{i}^{l} \leq \alpha_{i}^{u}<1, \quad 0<\beta_{i}^{l} \leq \beta_{i}^{u} .
\end{aligned}
$$

Here, we let $Z, Z^{+}$denote the sets of all integers, nonnegative integers, respectively, and use the notations: $f^{u}=\sup _{k \in Z}\{f(k)\}, f^{l}=\inf _{k \in Z}\{f(k)\}$, for any bounded sequence $\{f(k)\}$ defined on $Z$. conditions:

Let $\tau=\max \left\{\tau_{i}, \sigma_{i}, \rho_{i}, i=1,2\right\}$, we consider system (1.4) with the following initial

$$
\begin{gathered}
x_{i}(\theta)=\phi_{i}(\theta), \quad \theta \in[-\tau, 0] \cap Z, \phi_{i}(0)>0, \\
u_{i}(\theta)=\varphi_{i}(\theta), \quad \theta \in[-\tau, 0] \cap Z, \varphi_{i}(0)>0, i=1,2 .
\end{gathered}
$$

One can easily show that the solutions of system (1.4) with initial condition (1.5) are defined and remain positive for $k \in Z^{+}$.

The principle aim of this paper is to study the dynamic behaviors of system (1.4), such as permanence, global attractivity, and existence of a unique globally attractive positive almost periodic solution of the system. To the best of our knowledge, no work has been done for the nonautonomous difference system (1.4).

The organization of this paper is as follows. In the next section, we introduce some definitions and several useful lemmas. In Section 3, we explore the permanent property of system (1.4). We study globally attractive property of system (1.4) in Section 4 and the almost periodic property of system (1.4) in Section 5. Finally, the conclusion ends with brief remarks.

\section{Preliminaries}

In this section, we will introduce some basic definitions and several useful lemmas.

Definition 2.1. System (1.4) is said to be permanent, if there are positive constants $m_{i}$ and $M_{i}$, such that for each positive solution $\left(x_{1}(k), x_{2}(k), u_{1}(k), u_{2}(k)\right)^{T}$ of system (1.4) satisfies

$$
\begin{gathered}
m_{i} \leq \liminf _{k \rightarrow+\infty} x_{i}(\mathrm{k}) \leq \limsup _{k \rightarrow+\infty} x_{i}(k) \leq M_{i}, \\
n_{i} \leq \liminf _{k \rightarrow+\infty} u_{i}(k) \leq \limsup _{k \rightarrow+\infty} u_{i}(k) \leq N_{i}, \quad i=1,2 .
\end{gathered}
$$


Definition 2.2. Suppose that $X(k)=\left(x_{1}(k), x_{2}(k), u_{1}(k), u_{2}(k)\right)^{T}$ is any solution of system (1.4). $X(k)$ is said to be a strictly positive solution in $Z$ if for $k \in Z$ and $i=1,2$ such that

$$
0<\inf _{k \in Z} x_{i}(k) \leq \sup _{k \in Z} x_{i}(k)<\infty, \quad 0<\inf _{k \in Z} u_{i}(k) \leq \sup _{k \in Z} u_{i}(k)<\infty
$$

Definition 2.3 (see [16]). A sequence $x: Z \rightarrow R$ is called an almost periodic sequence if the $\varepsilon$-translation set of $x$

$$
E\{\varepsilon, x\}=\{\tau \in Z:|x(k+\tau)-x(k)|<\varepsilon, \forall k \in Z\}
$$

is a relatively dense set in $Z$ for all $\varepsilon>0$; that is, for any given $\varepsilon>0$, there exists an integer $l(\varepsilon)>0$ such that each interval of length $l(\varepsilon)$ contains an integer $\tau \in E\{\varepsilon, x\}$ with

$$
|x(k+\tau)-x(k)|<\varepsilon, \quad \forall k \in Z,
$$

$\tau$ is called an $\varepsilon$-translation number of $x(k)$.

Definition 2.4 (see [17]). The hull of $f$, denoted by $H(f)$, is defined by

$$
H(f)=\left\{g(k, x): \lim _{n \rightarrow \infty} f\left(k+\tau_{n}, x\right)=g(k, x) \text { uniformly on } Z \times S\right\}
$$

for some sequence $\left\{\tau_{n}\right\}$, where $S$ is any compact set in $D$.

Lemma 2.5 (see [20]). Assume that $\{y(k)\}$ satisfies $y\left(k_{1}\right)>0$ and

$$
y(k+1) \leq y(k) \exp \{r(k)(1-a y(k))\}
$$

for $k \in\left[k_{1},+\infty\right)$, where $a$ is a positive constant and $k_{1} \in Z^{+}$. Then

$$
\limsup _{k \rightarrow+\infty} y(k) \leq \frac{1}{a r^{u}} \exp \left(r^{u}-1\right)
$$

Lemma 2.6 (see [20]). Assume that $\{y(k)\}$ satisfies $y\left(k_{2}\right)>0$ and

$$
y(k+1) \geq y(k) \exp \{r(k)(1-a y(k))\}
$$

for $k \in\left[k_{2},+\infty\right), \limsup _{k \rightarrow+\infty} y(k) \leq M$, where $a$ is a constant such that $a M>1$ and $k_{2} \in Z^{+}$. Then

$$
\liminf _{k \rightarrow+\infty} y(k) \geq \frac{1}{a} \exp \left(r^{u}(1-a M)\right)
$$


Lemma 2.7 (see [22]). Assume that $A>0$ and $y(0)>0$. Suppose that

$$
y(k+1) \leq A y(k)+B(k), \quad n=1,2, \ldots
$$

Then for any integer $m \leq k$,

$$
y(k) \leq A^{m} y(k-m)+\sum_{j=0}^{m-1} A^{j} B(k-j-1) .
$$

Especially, if $A<1$ and $B$ is bounded above with respect to $M$, then

$$
\limsup _{k \rightarrow+\infty} y(k) \leq \frac{M}{1-A}
$$

Lemma 2.8 (see [22]). Assume that $A>0$ and $y(0)>0$. Suppose that

$$
y(k+1) \geq A y(k)+B(k), \quad n=1,2, \ldots
$$

Then for any integer $m \leq k$,

$$
y(k) \geq A^{m} y(k-m)+\sum_{j=0}^{m-1} A^{j} B(k-j-1) .
$$

Especially, if $A<1$ and $B$ is bounded below with respect to $N$, then

$$
\liminf _{k \rightarrow+\infty} y(k) \geq \frac{N}{1-A}
$$

\section{Permanence}

In this section, we establish a permanent result for system (1.4).

Theorem 3.1. Assume that $\left(H_{1}\right)$ holds; assume further that

$$
\left(H_{2}\right) b^{l}>c^{u} / 2|h|
$$

holds. Then system (1.4) is permanent.

Proof. Let $X(k)=\left(x_{1}(k), x_{2}(k), u_{1}(k), u_{2}(k)\right)^{T}$ be any positive solution of system (1.4), from the first equation of system (1.4), it follows that

$$
x_{1}(k+1) \leq x_{1}(k) \exp [b(k)] \leq x_{1}(k) \exp \left[b^{u}\right] .
$$


It follows from (3.1) that

$$
\prod_{j=k-\tau_{1}}^{k-1} \frac{x_{1}(j+1)}{x_{1}(j)} \leq \prod_{j=k-\tau_{1}}^{k-1} \exp \left[b^{u}\right] \leq \exp \left[b^{u} \tau_{1}\right]
$$

which implies that

$$
x_{1}\left(k-\tau_{1}\right) \geq x_{1}(k) \exp \left[-b^{u} \tau_{1}\right]
$$

Substituting (3.3) into the first equation of (1.4), it immediately follows that

$$
\begin{aligned}
x_{1}(k+1) & \leq x_{1}(k) \exp \left[b(k)-a(k) x_{1}\left(k-\tau_{1}\right)\right] \\
& \leq x_{1}(k) \exp \left[b(k)-a(k) \exp \left[-b^{u} \tau_{1}\right] x_{1}(k)\right] .
\end{aligned}
$$

By applying Lemma 2.5 to (3.4), we have

$$
\limsup _{k \rightarrow+\infty} x_{1}(k) \leq \frac{1}{a^{l}} \exp \left[b^{u}\left(\tau_{1}+1\right)-1\right]=: M_{1}
$$

For any $\varepsilon>0$ small enough, it follows from (3.5) that there exists enough large $K_{1}$ such that for $k \geq K_{1}$,

$$
x_{1}(k) \leq M_{1}+\varepsilon
$$

From the second equation of system (1.4) it follows that

$$
\begin{gathered}
x_{2}(k+1) \leq x_{2}(k) \exp [g(k)] \leq x_{2}(k) \exp \left[g^{u}\right], \\
x_{2}(k+1) \leq x_{2}(k) \exp \left[g(k)-\frac{f(k)}{M_{1}+\varepsilon} x_{2}\left(k-\tau_{2}\right)\right], \quad \text { for } k \geq K_{1}+\tau .
\end{gathered}
$$

From (3.7), similar to the argument of (3.1), one has

$$
x_{2}(k+1) \leq x_{2}(k) \exp \left[g(k)-\frac{f(k)}{M_{1}+\varepsilon} \exp \left[-g^{u} \tau_{2}\right] x_{2}(k)\right] .
$$

By applying Lemma 2.5 to (3.8) again, we have

$$
\limsup _{k \rightarrow+\infty} x_{2}(k) \leq \frac{M_{1}+\varepsilon}{f^{l}} \exp \left[g^{u}\left(\tau_{2}+1\right)-1\right] .
$$

Setting $\varepsilon \rightarrow 0$ in the above inequality, we have

$$
\limsup _{k \rightarrow+\infty} x_{2}(k) \leq \frac{M_{1}}{f^{l}} \exp \left[g^{u}\left(\tau_{2}+1\right)-1\right]=: M_{2} .
$$


For any $\varepsilon>0$ small enough, it follows from (3.5) and (3.10) that there exists enough large $K_{2}>K_{1}+\tau$ such that for $i=1,2$ and $k \geq K_{2}$

$$
x_{i}(k) \leq M_{i}+\varepsilon
$$

For $k>K_{2}+\tau$, (3.11) combining with the third and fourth equations of system (1.4) leads to

$$
\Delta u_{i}(k) \leq-\alpha_{i}(k) u_{i}(k)+\beta_{i}(k)\left(M_{i}+\varepsilon\right), \quad i=1,2,
$$

that is,

$$
u_{i}(k+1) \leq\left(1-\alpha_{i}^{l}\right) u_{i}(k)+\beta_{i}^{u}\left(M_{i}+\varepsilon\right), \quad i=1,2
$$

By applying Lemma 2.7, it follows from (3.13) that

$$
\limsup _{k \rightarrow+\infty} u_{i}(k) \leq \frac{\beta_{i}^{u}\left(M_{i}+\varepsilon\right)}{\alpha_{i}^{l}}, \quad i=1,2
$$

Letting $\varepsilon \rightarrow 0$ in the above inequality, we have

$$
\limsup _{k \rightarrow+\infty} u_{i}(k) \leq \frac{\beta_{i}^{u} M_{i}}{\alpha_{i}^{l}}=: N_{i}, \quad i=1,2
$$

For any $\varepsilon>0$ small enough, it follows from (3.11) and (3.15) that there exists enough large $K_{3}>K_{2}+\tau$ such that for $i=1,2$ and $k \geq K_{3}$

$$
x_{i}(k) \leq M_{i}+\varepsilon, \quad u_{i}(k) \leq N_{i}+\varepsilon .
$$

Thus, from (3.16) and the first equation of system (1.4), it follows that

$$
\begin{aligned}
x_{1}(k+1) & \geq x_{1}(k) \exp \left[b^{l}-a^{u}\left(M_{1}+\varepsilon\right)-\frac{c^{u}}{2|h|}-d^{u}\left(N_{1}+\varepsilon\right)\right] \\
& =: x_{1}(k) \exp \left[D_{1 \varepsilon}\right], \quad \text { for } k \geq K_{3}+\tau
\end{aligned}
$$

where

$$
\begin{aligned}
D_{1 \varepsilon} & =b^{l}-a^{u}\left(M_{1}+\varepsilon\right)-\frac{c^{u}}{2|h|}-d^{u}\left(N_{1}+\varepsilon\right) \\
& \leq b^{l}-a^{u} M_{1}
\end{aligned}
$$




$$
\begin{aligned}
& \leq b^{l}-\frac{a^{u} \exp \left[b^{u}-1\right]}{a^{l} \exp \left[-b^{u} \tau_{1}\right]} \\
& \leq b^{l}-b^{u} \\
& \leq 0 .
\end{aligned}
$$

For any integer $\eta \leq k$, it follows from (3.17) that

$$
\prod_{j=k-\eta}^{k-1} \frac{x_{1}(j+1)}{x_{1}(j)} \geq \prod_{j=k-\eta}^{k-1} \exp \left[D_{1 \varepsilon}\right] \geq \exp \left[D_{1 \varepsilon} \eta\right]
$$

and hence

$$
x_{1}(\eta) \leq x_{1}(k) \exp \left[-(k-\eta) D_{1 \varepsilon}\right]
$$

From the third equation of system (1.4), we have

$$
\begin{aligned}
u_{1}(k+1) & \leq\left(1-\alpha_{1}^{l}\right) u_{1}(k)+\beta_{1}^{u} x_{1}\left(k-\rho_{1}\right) \\
& \leq\left(1-\alpha_{1}^{l}\right) u_{1}(k)+\beta_{1}^{u} \exp \left[-\rho_{1} D_{1 \varepsilon}\right] x_{1}(k) \\
& =A_{1} u_{1}(k)+B_{1}(k)
\end{aligned}
$$

where $A_{1}=1-\alpha_{1}^{l}$ and $B_{1}(k)=\beta_{1}^{u} \exp \left[-\rho_{1} D_{1 \varepsilon}\right] x_{1}(k)$. Therefore, for any $m \leq k-\tau$, by Lemma 2.7, (3.20), and (3.21), one has

$$
\begin{aligned}
u_{1}(k) & \leq A_{1}^{m} u_{1}(k-m)+\sum_{j=0}^{m-1} A_{1}^{j} B_{1}(k-j-1) \\
& \leq A_{1}^{m} u_{1}(k-m)+\sum_{j=0}^{m-1} A_{1}^{j} \beta_{1}^{u} \exp \left[-\rho_{1} D_{1 \varepsilon}\right] x_{1}(k-j-1) \\
& \leq A_{1}^{m} u_{1}(k-m)+x_{1}(k) \sum_{j=0}^{m-1} A_{1}^{j} \beta_{1}^{u} \exp \left[-\left(j+1+\rho_{1}\right) D_{1 \varepsilon}\right] .
\end{aligned}
$$

Since $\alpha_{1}(k) \in(0,1)$, we have $0<A_{1}<1$. So

$$
0 \leq A_{1}^{m} u_{1}(k-m) \leq A_{1}^{m}\left(N_{1}+\varepsilon\right) \longrightarrow 0, \quad \text { for } m \longrightarrow \infty
$$


By the conditions $\left(H_{1}\right)$, for any solution $\left(x_{1}(k), x_{2}(k), u_{1}(k), u_{2}(k)\right)^{T}$ of system (1.4), there exists a positive integer $M$ such that $d^{u} A_{1}^{m} u_{1}(k-m)<(1 / 2)\left(b^{l}-c^{u} / 2|h|\right)$ for $m>M$. In fact, we can choose $M=\max \left\{1,\left[\log _{A_{1}}\left(\left(b^{l}-c^{u} / 2|h|\right) / 2 d^{u} N_{1}\right)\right]+1\right\}$. Then we get

$$
\begin{aligned}
u_{1}(k) & \leq A_{1}^{M}\left(N_{1}+\varepsilon\right)+x_{1}(k) \sum_{j=0}^{M-1} A_{1}^{j} \beta_{1}^{u} \exp \left[-\left(j+1+\rho_{1}\right) D_{1 \varepsilon}\right] \\
& =A_{1}^{M}\left(N_{1}+\varepsilon\right)+F_{1 \varepsilon} x_{1}(k),
\end{aligned}
$$

where $F_{1 \varepsilon}=\sum_{j=0}^{M-1} A_{1}^{j} \beta_{1}^{u} \exp \left[-\left(j+1+\rho_{1}\right) D_{1 \varepsilon}\right]$.

Substituting (3.20) and (3.24) into the first equation of system (1.4), one has

$$
\begin{aligned}
x_{1}(k+1) \geq x_{1}(k) \exp \left[b(k)-a(k) x_{1}\left(k-\tau_{1}\right)-\frac{c(k)}{2|h|}-d(k) u_{1}\left(k-\sigma_{1}\right)\right] \\
\geq x_{1}(k) \exp \left[b(k)-\frac{c(k)}{2|h|}-d(k) A_{1}^{M}\left(N_{1}+\varepsilon\right)\right. \\
\left.\quad-\left(a(k) \exp \left[-\tau_{1} D_{1 \varepsilon}\right]+d(k) F_{1 \varepsilon} \exp \left[-\sigma_{1} D_{1 \varepsilon}\right]\right) x_{1}(k)\right] \\
=: x_{1}(k) \exp \left[S_{1 \varepsilon}(k)\left(1-\left(\frac{S_{2 \varepsilon}(k)}{S_{1 \varepsilon}(k)}\right) x_{1}(k)\right)\right],
\end{aligned}
$$

where

$$
\begin{gathered}
S_{1 \varepsilon}(k)=b(k)-\frac{c(k)}{2|h|}-d(k) A_{1}^{M}\left(N_{1}+\varepsilon\right), \\
S_{2 \varepsilon}(k)=a(k) \exp \left[-\tau_{1} D_{1 \varepsilon}\right]+d(k) F_{1 \varepsilon} \exp \left[-\sigma_{1} D_{1 \varepsilon}\right] .
\end{gathered}
$$

In particular, we have

$$
\begin{aligned}
\frac{S_{2 \varepsilon}^{u}}{S_{1 \varepsilon}^{l}} M_{1} & =\frac{a^{u} \exp \left[-\tau_{1} D_{1 \varepsilon}\right]+d^{u} F_{1 \varepsilon} \exp \left[-\sigma_{1} D_{1 \varepsilon}\right]}{b^{l}-c^{u} / 2|h|-d^{u} A_{1}^{M}\left(N_{1}+\varepsilon\right)} M_{1} \\
& \geq \frac{a^{u} \exp \left[-\tau_{1} D_{1 \varepsilon}\right]}{b^{l}} \frac{\exp \left[b^{u}\left(\tau_{1}+1\right)-1\right]}{a^{l}} \\
& \geq \frac{a^{u} \exp \left[-\tau_{1} b^{l}\right]}{b^{l}} \frac{\exp \left[b^{l}\left(\tau_{1}+1\right)-1\right]}{a^{u}} \\
& \geq \frac{\exp \left[b^{l}-1\right]}{b^{l}} \\
& >1,
\end{aligned}
$$


where we use the inequality $\exp (x-1)>x$ for $x>0$. By applying Lemma 2.6 to (3.25), it follows that

$$
\liminf _{k \rightarrow+\infty} x_{1}(k) \geq \frac{S_{1 \varepsilon}^{l}}{S_{2 \varepsilon}^{u}} \exp \left[S_{1 \varepsilon}^{u}\left(1-\frac{S_{2 \varepsilon}^{u}}{S_{1 \varepsilon}^{l}} M_{1}\right)\right]
$$

Setting $\varepsilon \rightarrow 0$ in the above inequality, we have

$$
\liminf _{k \rightarrow+\infty} x_{1}(k) \geq \frac{S_{1}^{l}}{S_{2}^{u}} \exp \left[S_{1}^{u}\left(1-\frac{S_{2}^{u}}{S_{1}^{l}} M_{1}\right)\right]=: m_{1}
$$

From (3.29) we know that there exists enough large $K_{4}>K_{3}+\tau$ such that for $k \geq K_{4}$,

$$
x_{1}(k) \geq m_{1}-\varepsilon .
$$

From (3.30) and the second equation of system (1.4), it follows that

$$
\begin{aligned}
x_{2}(k+1) & \geq x_{2}(k) \exp \left[g^{l}-\frac{f^{u}\left(M_{2}+\varepsilon\right)}{m_{1}-\varepsilon}-p^{u}\left(N_{2}+\varepsilon\right)\right] \\
& =: x_{2}(k) \exp \left[D_{2 \varepsilon}\right], \quad \text { for } k \geq K_{4}+\tau,
\end{aligned}
$$

where $D_{2 \varepsilon}=g^{l}-f^{u}\left(M_{2}+\varepsilon\right) /\left(m_{1}-\varepsilon\right)-p^{u}\left(N_{2}+\varepsilon\right) \leq 0$.

This implies for any integer $\eta \leq k$

$$
x_{2}(\eta) \leq x_{2}(k) \exp \left[-(k-\eta) D_{2 \varepsilon}\right] .
$$

From (3.32) and the fourth equation of system (1.4), by a procedure similar to the discussion of (3.21)-(3.24), we can verify that

$$
u_{2}(k) \leq A_{2}^{N}\left(N_{2}+\varepsilon\right)+F_{2 \varepsilon} x_{2}(k),
$$

where

$$
\begin{gathered}
A_{2}=1-\alpha_{2}^{l}, \quad F_{2 \varepsilon}=\sum_{j=0}^{N-1} A_{2}^{j} \beta_{2}^{u} \exp \left[-\left(j+1+\rho_{2}\right) D_{2 \varepsilon}\right], \\
N=\max \left\{1,\left[\log _{A_{2}}\left(\frac{g^{l}}{2 p^{u} N_{2}}\right)\right]+1\right\} .
\end{gathered}
$$


Substituting (3.32) and (3.33) into the second equation of system (1.4), one has

$$
\begin{aligned}
x_{2}(k+1) \geq x_{2}(k) \exp \left[g(k)-\frac{f(k)}{m_{1}-\varepsilon} x_{2}\left(k-\tau_{2}\right)-p(k) u_{2}\left(k-\sigma_{2}\right)\right] \\
\geq x_{2}(k) \exp \left[g(k)-p(k) A_{2}^{N}\left(N_{2}+\varepsilon\right)\right. \\
\left.\quad \quad-\left(\frac{f(k)}{m_{1}} \exp \left[-\tau_{2} D_{2 \varepsilon}\right]+p(k) F_{2 \varepsilon} \exp \left[-\sigma_{2} D_{2 \varepsilon}\right]\right) x_{2}(k)\right] \\
=: x_{2}(k) \exp \left[S_{3 \varepsilon}(k)\left(1-\left(\frac{S_{4 \varepsilon}(k)}{S_{3 \varepsilon}(k)}\right) x_{2}(k)\right)\right],
\end{aligned}
$$

where

$$
\begin{gathered}
S_{3 \varepsilon}(k)=g(k)-p(k) A_{2}^{N}\left(N_{2}+\varepsilon\right), \\
S_{4 \varepsilon}(k)=\frac{f(k)}{m_{1}} \exp \left[-\tau_{2} D_{2 \varepsilon}\right]+p(k) F_{2 \varepsilon} \exp \left[-\sigma_{2} D_{2 \varepsilon}\right] .
\end{gathered}
$$

In particular, we have

$$
\begin{aligned}
\frac{S_{4 \varepsilon}^{u}}{S_{3 \varepsilon}^{l}} M_{2} & =\frac{\left(f^{u} / m_{1}\right) \exp \left[-\tau_{2} D_{2 \varepsilon}\right]+p^{u} F_{2} \exp \left[-\sigma_{2} D_{2 \varepsilon}\right]}{g^{l}-p^{u} A_{2}^{N}\left(N_{2}+\varepsilon\right)} M_{2} \\
& \geq \frac{\left(f^{u} / m_{1}\right) \exp \left[-\tau_{2} D_{2 \varepsilon}\right]}{g^{l}} \frac{M_{1} \exp \left[g^{u}\left(\tau_{2}+1\right)-1\right]}{f^{l}} \\
& \geq \frac{\left(f^{u} / m_{1}\right) \exp \left[-\tau_{2} g^{l}\right]}{g^{l}} \frac{M_{1} \exp \left[g^{l}\left(\tau_{2}+1\right)-1\right]}{f^{u}} \\
& \geq \frac{\exp \left[g^{l}-1\right]}{g^{l}} \\
& >1 .
\end{aligned}
$$

By applying Lemma 2.6 to (3.35) again, it follows that

$$
\liminf _{k \rightarrow+\infty} x_{2}(k) \geq \frac{S_{3 \varepsilon}^{l}}{S_{4 \varepsilon}^{u}} \exp \left[S_{3 \varepsilon}^{u}\left(1-\frac{S_{4 \varepsilon}^{u}}{S_{3 \varepsilon}^{l}} M_{2}\right)\right] .
$$

Setting $\varepsilon \rightarrow 0$ in the above inequality, we have

$$
\liminf _{k \rightarrow+\infty} x_{2}(k) \geq \frac{S_{3}^{l}}{S_{4}^{u}} \exp \left[S_{3}^{u}\left(1-\frac{S_{4}^{u}}{S_{3}^{l}} M_{2}\right)\right]=: m_{2}
$$


From (3.29) and (3.39) we know that there exists enough large $K_{5}>K_{4}+\tau$ such that for $k \geq K_{5}$,

$$
x_{i}(k) \geq m_{i}-\varepsilon
$$

For $k>K_{5}+\tau$, (3.40) combining with the third and fourth equations of system (1.4) produces

$$
u_{i}(k+1) \geq\left(1-\alpha_{i}^{u}\right) u_{i}(k)+\beta_{i}^{l}\left(m_{i}-\varepsilon\right), \quad i=1,2 .
$$

By applying Lemma 2.8, it follows from (3.41) that

$$
\liminf _{k \rightarrow+\infty} u_{i}(k) \geq \frac{\beta_{i}^{l}\left(m_{i}-\varepsilon\right)}{\alpha_{i}^{u}}, \quad i=1,2
$$

Setting $\varepsilon \rightarrow 0$ in the above inequality, we have

$$
\liminf _{k \rightarrow+\infty} u_{i}(k) \geq \frac{\beta_{i}^{l} m_{i}}{\alpha_{i}^{u}}=: n_{i}, \quad i=1,2
$$

Consequently, combining (3.5), (3.10), (3.15), (3.29), (3.39) with (3.43), system (1.4) is permanent. This completes the proof of Theorem 3.1.

\section{Global Attractivity}

Firstly, we prove two lemmas which will be useful to our main result.

Lemma 4.1. For any two positive solutions $\left(x_{1}(k), x_{2}(k), u_{1}(k), u_{2}(k)\right)^{T}$ and $\left(y_{1}(k), y_{2}(k), v_{1}(k)\right.$, $\left.v_{2}(k)\right)^{T}$ of system (1.4), one has

$$
\begin{aligned}
\ln \frac{x_{1}(k+1)}{y_{1}(k+1)} \\
=\ln \frac{x_{1}(k)}{y_{1}(k)}-a(k)\left[x_{1}(k)-y_{1}(k)\right] \\
\quad-d(k)\left[u_{1}\left(k-\sigma_{1}\right)-v_{1}\left(k-\sigma_{1}\right)\right]+E_{1}(k)\left[x_{1}(k)-y_{1}(k)\right]+E_{2}(k)\left[x_{2}(k)-y_{2}(k)\right]
\end{aligned}
$$




$$
\begin{aligned}
+a(k) \sum_{s=k-\tau_{1}}^{k-1}\left\{P_{1}(s)[\right. & b(s)-a(s) y_{1}\left(s-\tau_{1}\right) \\
& \left.-\frac{c(s) y_{1}(s) y_{2}(s)}{h^{2} y_{2}^{2}(s)+y_{1}^{2}(s)}-d(s) v_{1}\left(s-\sigma_{1}\right)\right]\left[x_{1}(s)-y_{1}(s)\right] \\
+ & x_{1}(s) P_{2}(s)\left[-a(s)\left[x_{1}\left(s-\tau_{1}\right)-y_{1}\left(s-\tau_{1}\right)\right]\right. \\
& -d(s)\left[u_{1}\left(s-\sigma_{1}\right)-v_{1}\left(s-\sigma_{1}\right)\right] \\
& \left.\left.+E_{1}(s)\left[x_{1}(s)-y_{1}(s)\right]+E_{2}(s)\left[x_{2}(s)-y_{2}(s)\right]\right]\right\},
\end{aligned}
$$

where

$$
\begin{aligned}
& E_{1}(s)=\frac{c(s) y_{1}(s) y_{2}(s)\left[x_{1}(s)+y_{1}(s)\right]}{\left[h^{2} x_{2}^{2}(s)+x_{1}^{2}(s)\right]\left[h^{2} y_{2}^{2}(s)+y_{1}^{2}(s)\right]}-\frac{c(s) x_{2}(s)}{h^{2} x_{2}^{2}(s)+x_{1}^{2}(s)}, \\
& E_{2}(s)= \frac{h^{2} c(s) y_{1}(s) y_{2}(s)\left[x_{2}(s)+y_{2}(s)\right]}{\left[h^{2} x_{2}^{2}(s)+x_{1}^{2}(s)\right]\left[h^{2} y_{2}^{2}(s)+y_{1}^{2}(s)\right]}-\frac{c(s) y_{1}(s)}{h^{2} x_{2}^{2}(s)+x_{1}^{2}(s)}, \\
& P_{1}(s)=\exp \left\{\theta_{1}(s)\left[b(s)-a(s) y_{1}\left(s-\tau_{1}\right)-\frac{c(s) y_{1}(s) y_{2}(s)}{h^{2} y_{2}^{2}(s)+y_{1}^{2}(s)}-d(s) v_{1}\left(s-\sigma_{1}\right)\right]\right\}, \\
& P_{2}(s)=\exp \left\{\theta_{2}(s)\left[b(s)-a(s) x_{1}\left(s-\tau_{1}\right)-\frac{c(s) x_{1}(s) x_{2}(s)}{h^{2} x_{2}^{2}(s)+x_{1}^{2}(s)}-d(s) u_{1}\left(s-\sigma_{1}\right)\right]\right. \\
&\left.\quad+\left(1-\theta_{2}(s)\right)\left[b(s)-a(s) y_{1}\left(s-\tau_{1}\right)-\frac{c(s) y_{1}(s) y_{2}(s)}{h^{2} y_{2}^{2}(s)+y_{1}^{2}(s)}-d(s) v_{1}\left(s-\sigma_{1}\right)\right]\right\},
\end{aligned}
$$

Proof. It follows from the first equation of system (1.4) that

$$
\begin{aligned}
\ln & \frac{x_{1}(k+1)}{y_{1}(k+1)}-\ln \frac{x_{1}(k)}{y_{1}(k)} \\
= & \ln \frac{x_{1}(k+1)}{x_{1}(k)}-\ln \frac{y_{1}(k+1)}{y_{1}(k)} \\
= & {\left[b(k)-a(k) x_{1}\left(k-\tau_{1}\right)-\frac{c(k) x_{1}(k) x_{2}(k)}{h^{2} x_{2}^{2}(k)+x_{1}^{2}(k)}-d(k) u_{1}\left(k-\sigma_{1}\right)\right] } \\
& -\left[b(k)-a(k) y_{1}\left(k-\tau_{1}\right)-\frac{c(k) y_{1}(k) y_{2}(k)}{h^{2} y_{2}^{2}(k)+y_{1}^{2}(k)}-d(k) v_{1}\left(k-\sigma_{1}\right)\right]
\end{aligned}
$$




$$
\begin{aligned}
= & -a(k)\left[x_{1}\left(k-\tau_{1}\right)-y_{1}\left(k-\tau_{1}\right)\right]-d(k)\left[u_{1}\left(k-\sigma_{1}\right)-v_{1}\left(k-\sigma_{1}\right)\right] \\
& -\frac{c(k) x_{1}(k) x_{2}(k)}{h^{2} x_{2}^{2}(k)+x_{1}^{2}(k)}+\frac{c(k) y_{1}(k) y_{2}(k)}{h^{2} y_{2}^{2}(k)+y_{1}^{2}(k)} \\
= & -a(k)\left[x_{1}(k)-y_{1}(k)\right]-d(k)\left[u_{1}\left(k-\sigma_{1}\right)-v_{1}\left(k-\sigma_{1}\right)\right] \\
& +E_{1}(k)\left[x_{1}(k)-y_{1}(k)\right]+E_{2}(k)\left[x_{2}(k)-y_{2}(k)\right] \\
& +a(k)\left\{\left[x_{1}(k)-y_{1}(k)\right]-\left[x_{1}\left(k-\tau_{1}\right)-y_{1}\left(k-\tau_{1}\right)\right]\right\}
\end{aligned}
$$

where

$$
\begin{aligned}
& E_{1}(k)=\frac{c(k) y_{1}(k) y_{2}(k)\left[x_{1}(k)+y_{1}(k)\right]}{\left[h^{2} x_{2}^{2}(k)+x_{1}^{2}(k)\right]\left[h^{2} y_{2}^{2}(k)+y_{1}^{2}(k)\right]}-\frac{c(k) x_{2}(k)}{h^{2} x_{2}^{2}(k)+x_{1}^{2}(k)}, \\
& E_{2}(k)=\frac{h^{2} c(k) y_{1}(k) y_{2}(k)\left[x_{2}(k)+y_{2}(k)\right]}{\left[h^{2} x_{2}^{2}(k)+x_{1}^{2}(k)\right]\left[h^{2} y_{2}^{2}(k)+y_{1}^{2}(k)\right]}-\frac{c(k) y_{1}(k)}{h^{2} x_{2}^{2}(k)+x_{1}^{2}(k)} .
\end{aligned}
$$

Hence

$$
\begin{aligned}
\ln \frac{x_{1}(k+1)}{y_{1}(k+1)}= & \ln \frac{x_{1}(k)}{y_{1}(k)}-a(k)\left[x_{1}(k)-y_{1}(k)\right]-d(k)\left[u_{1}\left(k-\sigma_{1}\right)-v_{1}\left(k-\sigma_{1}\right)\right] \\
& +E_{1}(k)\left[x_{1}(k)-y_{1}(k)\right]+E_{2}(k)\left[x_{2}(k)-y_{2}(k)\right] \\
& +a(k)\left\{\left[x_{1}(k)-x_{1}\left(k-\tau_{1}\right)\right]-\left[y_{1}(k)-y_{1}\left(k-\tau_{1}\right)\right]\right\} .
\end{aligned}
$$

Since

$$
\begin{aligned}
& {\left[x_{1}(k)-x_{1}\left(k-\tau_{1}\right)\right]-\left[y_{1}(k)-y_{1}\left(k-\tau_{1}\right)\right]} \\
& =\sum_{s=k-\tau_{1}}^{k-1}\left[x_{1}(s+1)-x_{1}(s)\right]-\sum_{s=k-\tau_{1}}^{k-1}\left[y_{1}(s+1)-y_{1}(s)\right] \\
& =\sum_{s=k-\tau_{1}}^{k-1}\left\{\left[x_{1}(s+1)-y_{1}(s+1)\right]-\left[x_{1}(s)-y_{1}(s)\right]\right\}, \\
& {\left[x_{1}(s+1)-y_{1}(s+1)\right]-\left[x_{1}(s)-y_{1}(s)\right]} \\
& =\left\{x_{1}(s) \exp \left[b(s)-a(s) x_{1}\left(s-\tau_{1}\right)-\frac{c(s) x_{1}(s) x_{2}(s)}{h^{2} x_{2}^{2}(s)+x_{1}^{2}(s)}-d(s) u_{1}\left(s-\sigma_{1}\right)\right]\right. \\
& \left.\quad-y_{1}(s) \exp \left[b(s)-a(s) y_{1}\left(s-\tau_{1}\right)-\frac{c(s) y_{1}(s) y_{2}(s)}{h^{2} y_{2}^{2}(s)+y_{1}^{2}(s)}-d(s) v_{1}\left(s-\sigma_{1}\right)\right]\right\} \\
& \quad-\left[x_{1}(s)-y_{1}(s)\right]
\end{aligned}
$$


Abstract and Applied Analysis

$$
\begin{aligned}
=\left[x_{1}(s)-y_{1}(s)\right]\left\{\exp \left[b(s)-a(s) y_{1}\left(s-\tau_{1}\right)-\frac{c(s) y_{1}(s) y_{2}(s)}{h^{2} y_{2}^{2}(s)+y_{1}^{2}(s)}-d(s) v_{1}\left(s-\sigma_{1}\right)\right]-1\right\} \\
+x_{1}(s)\left\{\exp \left[b(s)-a(s) x_{1}\left(s-\tau_{1}\right)-\frac{c(s) x_{1}(s) x_{2}(s)}{h^{2} x_{2}^{2}(s)+x_{1}^{2}(s)}-d(s) u_{1}\left(s-\sigma_{1}\right)\right]\right. \\
\left.-\exp \left[b(s)-a(s) y_{1}\left(s-\tau_{1}\right)-\frac{c(s) y_{1}(s) y_{2}(s)}{h^{2} y_{2}^{2}(s)+y_{1}^{2}(s)}-d(s) v_{1}\left(s-\sigma_{1}\right)\right]\right\} .
\end{aligned}
$$

By the mean value theorem, one has

$$
\begin{aligned}
& {\left[x_{1}(s+1)-y_{1}(s+1)\right]-\left[x_{1}(s)-y_{1}(s)\right]} \\
& =\left[x_{1}(s)-y_{1}(s)\right] P_{1}(s)\left[b(s)-a(s) y_{1}\left(s-\tau_{1}\right)-\frac{c(s) y_{1}(s) y_{2}(s)}{h^{2} y_{2}^{2}(s)+y_{1}^{2}(s)}-d(s) v_{1}\left(s-\sigma_{1}\right)\right] \\
& +x_{1}(s) P_{2}(s)\left[-a(s)\left[x_{1}\left(s-\tau_{1}\right)-y_{1}\left(s-\tau_{1}\right)\right]-d(s)\left[u_{1}\left(s-\sigma_{1}\right)-v_{1}\left(s-\sigma_{1}\right)\right]\right. \\
& \left.\quad-\frac{c(s) x_{1}(s) x_{2}(s)}{h^{2} x_{2}^{2}(s)+x_{1}^{2}(s)}+\frac{c(s) y_{1}(s) y_{2}(s)}{h^{2} y_{2}^{2}(s)+y_{1}^{2}(s)}\right] \\
& =\left[x_{1}(s)-y_{1}(s)\right] P_{1}(s)\left[b(s)-a(s) y_{1}\left(s-\tau_{1}\right)-\frac{c(s) y_{1}(s) y_{2}(s)}{h^{2} y_{2}^{2}(s)+y_{1}^{2}(s)}-d(s) v_{1}\left(s-\sigma_{1}\right)\right] \\
& +x_{1}(s) P_{2}(s)\left[-a(s)\left[x_{1}\left(s-\tau_{1}\right)-y_{1}\left(s-\tau_{1}\right)\right]-d(s)\left[u_{1}\left(s-\sigma_{1}\right)-v_{1}\left(s-\sigma_{1}\right)\right]\right. \\
& \left.+E_{1}(s)\left[x_{1}(s)-y_{1}(s)\right]+E_{2}(s)\left[x_{2}(s)-y_{2}(s)\right]\right] .
\end{aligned}
$$

Combining (4.6) with (4.8), we can easily obtain (4.1). The proof of Lemma 4.1 is completed.

Lemma 4.2. For any two positive solutions $\left(x_{1}(k), x_{2}(k), u_{1}(k), u_{2}(k)\right)^{T}$ and $\left(y_{1}(k), y_{2}(k), v_{1}(k)\right.$, $\left.v_{2}(k)\right)^{T}$ of system (1.4), one has

$$
\begin{aligned}
& \ln \frac{x_{2}(k+1)}{y_{2}(k+1)} \\
& =\ln \frac{x_{2}(k)}{y_{2}(k)}-\frac{f(k)}{x_{1}\left(k-\tau_{2}\right)}\left[x_{2}(k)-y_{2}(k)\right]+\frac{f(k) y_{2}\left(k-\tau_{2}\right)}{x_{1}\left(k-\tau_{2}\right) y_{1}\left(k-\tau_{2}\right)}\left[x_{1}\left(k-\tau_{2}\right)-y_{1}\left(k-\tau_{2}\right)\right] \\
& \quad-p(k)\left[u_{2}\left(k-\sigma_{2}\right)-v_{2}\left(k-\sigma_{2}\right)\right]
\end{aligned}
$$




$$
\begin{aligned}
+\frac{f(k)}{x_{1}\left(k-\tau_{2}\right)} \sum_{s=k-\tau_{2}}^{k-1}\left\{Q_{1}(s)[g(s)-\right. & \left.f(s) \frac{y_{2}\left(s-\tau_{2}\right)}{y_{1}\left(s-\tau_{2}\right)}-p(s) v_{2}\left(s-\sigma_{2}\right)\right]\left[x_{2}(s)-y_{2}(s)\right] \\
+x_{2}(s) Q_{2}(s)[ & -\frac{f(s)}{x_{1}\left(s-\tau_{2}\right)}\left[x_{2}\left(s-\tau_{2}\right)-y_{2}\left(s-\tau_{2}\right)\right] \\
& +\frac{f(s) y_{2}\left(s-\tau_{2}\right)}{x_{1}\left(s-\tau_{2}\right) y_{1}\left(s-\tau_{2}\right)}\left[x_{1}\left(s-\tau_{2}\right)-y_{1}\left(s-\tau_{2}\right)\right] \\
& \left.\left.-p(k)\left[u_{2}\left(s-\sigma_{2}\right)-v_{2}\left(s-\sigma_{2}\right)\right]\right]\right\}
\end{aligned}
$$

where

$$
\begin{aligned}
Q_{1}(s)=\exp \left\{\varphi_{1}(s)\left[g(s)-f(s) \frac{y_{2}\left(s-\tau_{2}\right)}{y_{1}\left(s-\tau_{2}\right)}-p(s) v_{2}\left(s-\sigma_{2}\right)\right]\right\} \\
Q_{2}(s)=\exp \left\{\varphi_{2}(s)\left[g(s)-f(s) \frac{x_{2}\left(s-\tau_{2}\right)}{x_{1}\left(s-\tau_{2}\right)}-p(s) u_{2}\left(s-\sigma_{2}\right)\right]\right. \\
\left.+\left(1-\varphi_{2}(s)\right)\left[g(s)-f(s) \frac{y_{2}\left(s-\tau_{2}\right)}{y_{1}\left(s-\tau_{2}\right)}-p(s) v_{2}\left(s-\sigma_{2}\right)\right]\right\}, \quad \varphi_{1}(s), \varphi_{2}(s) \in(0,1) .
\end{aligned}
$$

Proof. The proofs of Lemma 4.2 are very similar to those of Lemma 4.1. So we omit the detail here.

Now we are in the position of stating the main result on the global attractivity of system (1.4).

Theorem 4.3. In addition to $\left(H_{1}\right)-\left(H_{2}\right)$, assume further that

$$
\begin{gathered}
\left(H_{3}\right) \text { there exist positive constants } s_{i}, \omega_{i}, i=1,2 \text { such that } \\
\alpha=: \min \left(s_{1} \mu_{1}-s_{2} \mu_{2}-\omega_{1} \beta_{1}^{u}, s_{2} v_{1}-s_{1} v_{2}-\omega_{2} \beta_{2}^{u}, \omega_{1} \alpha_{1}^{l}-s_{1} \varrho_{1}, \omega_{2} \alpha_{2}^{l}-s_{2} \varrho_{2}\right)>0
\end{gathered}
$$

holds, where $\mu_{1}, \mu_{2}, v_{1}, v_{2}, Q_{1}, Q_{2}$ are defined by (4.37). Then for any two positive solutions $\left(x_{1}(k)\right.$, $\left.x_{2}(k), u_{1}(k), u_{2}(k)\right)^{T}$ and $\left(y_{1}(k), y_{2}(k), v_{1}(k), v_{2}(k)\right)^{T}$ of system $(1.4)$, one has

$$
\lim _{k \rightarrow+\infty}\left|x_{i}(k)-y_{i}(k)\right|=0, \quad \lim _{k \rightarrow+\infty}\left|u_{i}(k)-v_{i}(k)\right|=0, \quad i=1,2
$$


Proof. For two arbitrary nontrivial solutions $\left(x_{1}(k), x_{2}(k), u_{1}(k), u_{2}(k)\right)^{T}$ and $\left(y_{1}(k), y_{2}(k)\right.$, $\left.v_{1}(k), v_{2}(k)\right)^{T}$ of system (1.4), we have from Theorem 3.1 that there exist positive constants $k_{0}$ and $M_{i}, N_{i}, m_{i}, n_{i}(i=1,2)$ such that for all $k \geq k_{0}$ and $i=1,2$

$$
\begin{array}{ll}
m_{i} \leq x_{i}(k) \leq M_{i}, & n_{i} \leq u_{i}(k) \leq N_{i} \\
m_{i} \leq y_{i}(k) \leq M_{i}, & n_{i} \leq v_{i}(k) \leq N_{i} .
\end{array}
$$

Firstly, we define

$$
V_{11}(k)=\left|\ln x_{1}(k)-\ln y_{1}(k)\right|
$$

From (4.1), we have

$$
\begin{array}{r}
\left|\ln \frac{x_{1}(k+1)}{y_{1}(k+1)}\right| \leq\left|\ln \frac{x_{1}(k)}{y_{1}(k)}-a(k)\left[x_{1}(k)-y_{1}(k)\right]\right|+d(k)\left|u_{1}\left(k-\sigma_{1}\right)-v_{1}\left(k-\sigma_{1}\right)\right| \\
+E_{1}(k)\left|x_{1}(k)-y_{1}(k)\right|+E_{2}(k)\left|x_{2}(k)-y_{2}(k)\right| \\
+a(k) \sum_{s=k-\tau_{1}}^{k-1}\left\{\left[P_{1}(s) G_{1}(s)+x_{1}(s) P_{2}(s) E_{1}(s)\right]\left|x_{1}(s)-y_{1}(s)\right|\right. \\
+x_{1}(s) P_{2}(s)\left[a(s)\left|x_{1}\left(s-\tau_{1}\right)-y_{1}\left(s-\tau_{1}\right)\right|\right. \\
+d(s)\left|u_{1}\left(s-\sigma_{1}\right)-v_{1}\left(s-\sigma_{1}\right)\right| \\
\left.\left.+E_{2}(s)\left|x_{2}(s)-y_{2}(s)\right|\right]\right\}
\end{array}
$$

where

$$
G_{1}(s)=b(s)+a(s) y_{1}\left(s-\tau_{1}\right)+\frac{c(s) y_{1}(s) y_{2}(s)}{h^{2} y_{2}^{2}(s)+y_{1}^{2}(s)}+d(s) v_{1}\left(s-\sigma_{1}\right)
$$

By the mean value theorem, we have

$$
x_{1}(k)-y_{1}(k)=\exp \left[\ln x_{1}(k)\right]-\exp \left[\ln y_{1}(k)\right]=\xi_{1}(k) \ln \frac{x_{1}(k)}{y_{1}(k)}
$$

that is,

$$
\ln \frac{x_{1}(k)}{y_{1}(k)}=\frac{1}{\xi_{1}(k)}\left[x_{1}(k)-y_{1}(k)\right]
$$


where $\xi_{1}(k)$ lies between $x_{1}(k)$ and $y_{1}(k)$. So, we have

$$
\begin{aligned}
& \left|\ln \frac{x_{1}(k)}{y_{1}(k)}-a(k)\left[x_{1}(k)-y_{1}(k)\right]\right| \\
& \quad=\left|\ln \frac{x_{1}(k)}{y_{1}(k)}\right|-\left|\ln \frac{x_{1}(k)}{y_{1}(k)}\right|+\left|\ln \frac{x_{1}(k)}{y_{1}(k)}-a(k)\left[x_{1}(k)-y_{1}(k)\right]\right| \\
& =\left|\ln \frac{x_{1}(k)}{y_{1}(k)}\right|-\left[\frac{1}{\xi_{1}(k)}-\left|\frac{1}{\xi_{1}(k)}-a(k)\right|\right]\left|x_{1}(k)-y_{1}(k)\right| .
\end{aligned}
$$

For $k>k_{0}+\tau$, it follows that

$$
\begin{aligned}
\Delta V_{11} \leq & -\left[\frac{1}{\xi_{1}(k)}-\left|\frac{1}{\xi_{1}(k)}-a(k)\right|\right]\left|x_{1}(k)-y_{1}(k)\right| \\
& +d(k)\left|u_{1}\left(k-\sigma_{1}\right)-v_{1}\left(k-\sigma_{1}\right)\right| \\
& +E_{1}(k)\left|x_{1}(k)-y_{1}(k)\right|+E_{2}(k)\left|x_{2}(k)-y_{2}(k)\right| \\
& +a(k) \sum_{s=k-\tau_{1}}^{k-1}\left\{\left[P_{1}(s) G_{1}(s)+M_{1} P_{2}(s) E_{1}(s)\right]\left|x_{1}(s)-y_{1}(s)\right|\right. \\
& +M_{1} P_{2}(s)\left[a(s)\left|x_{1}\left(s-\tau_{1}\right)-y_{1}\left(s-\tau_{1}\right)\right|\right. \\
& +d(s)\left|u_{1}\left(s-\sigma_{1}\right)-v_{1}\left(s-\sigma_{1}\right)\right| \\
& \left.\left.+E_{2}(s)\left|x_{2}(s)-y_{2}(s)\right|\right]\right\} .
\end{aligned}
$$

Secondly, we define

$$
\begin{aligned}
V_{12}(k)= & \sum_{s=k-\sigma_{1}}^{k-1} d\left(s+\sigma_{1}\right)\left|u_{1}(s)-v_{1}(s)\right| \\
+\sum_{s=k}^{k-1+\tau_{1}} a(s) \sum_{u=s-\tau_{1}}^{k-1}\{ & {\left[P_{1}(u) G_{1}(u)+M_{1} P_{2}(u) E_{1}(u)\right]\left|x_{1}(u)-y_{1}(u)\right| } \\
+M_{1} P_{2}(u)\left[a(u)\left|x_{1}\left(u-\tau_{1}\right)-y_{1}\left(u-\tau_{1}\right)\right|\right. & \\
& +d(u)\left|u_{1}\left(u-\sigma_{1}\right)-v_{1}\left(u-\sigma_{1}\right)\right| \\
& \left.\left.+E_{2}(u)\left|x_{2}(u)-y_{2}(u)\right|\right]\right\} .
\end{aligned}
$$


Then

$$
\begin{gathered}
\Delta V_{12}=d\left(k+\sigma_{1}\right)\left|u_{1}(k)-v_{1}(k)\right|-d(k)\left|u_{1}\left(k-\sigma_{1}\right)-v_{1}\left(k-\sigma_{1}\right)\right| \\
+\sum_{s=k+1}^{k+\tau_{1}} a(s)\left\{\left[P_{1}(k) G_{1}(k)+M_{1} P_{2}(k) E_{1}(k)\right]\left|x_{1}(k)-y_{1}(k)\right|\right. \\
+M_{1} P_{2}(k)\left[a(k)\left|x_{1}\left(k-\tau_{1}\right)-y_{1}\left(k-\tau_{1}\right)\right|\right. \\
\left.\left.+d(k)\left|u_{1}\left(k-\sigma_{1}\right)-v_{1}\left(k-\sigma_{1}\right)\right|+E_{2}(k)\left|x_{2}(k)-y_{2}(k)\right|\right]\right\} \\
-a(k) \sum_{u=s-\tau_{1}}^{k-1}\left\{\left[P_{1}(u) G_{1}(u)+M_{1} P_{2}(u) E_{1}(u)\right]\left|x_{1}(u)-y_{1}(u)\right|\right. \\
+M_{1} P_{2}(u)\left[a(u)\left|x_{1}\left(u-\tau_{1}\right)-y_{1}\left(u-\tau_{1}\right)\right|\right. \\
\left.\left.+d(u)\left|u_{1}\left(u-\sigma_{1}\right)-v_{1}\left(u-\sigma_{1}\right)\right|+E_{2}(u)\left|x_{2}(u)-y_{2}(u)\right|\right]\right\} .
\end{gathered}
$$

Thirdly, we define

$$
\begin{aligned}
V_{13}(k)= & M_{1} \sum_{l=k-\tau_{1}}^{k-1} P_{2}\left(l+\tau_{1}\right) a\left(l+\tau_{1}\right)\left|x_{1}(l)-y_{1}(l)\right| \sum_{s=l+\tau_{1}+1}^{l+2 \tau_{1}} a(s) \\
& +M_{1} \sum_{l=k-\sigma_{1}}^{k-1} P_{2}\left(l+\sigma_{1}\right) d\left(l+\sigma_{1}\right)\left|u_{1}(l)-v_{1}(l)\right| \sum_{s=l+\sigma_{1}+1}^{l+\sigma_{1}+\tau_{1}} a(s) .
\end{aligned}
$$

By a simple calculation, it follows that

$$
\begin{aligned}
\Delta V_{13}= & \sum_{s=k+\tau_{1}+1}^{k+2 \tau_{1}} a(s) M_{1} P_{2}\left(k+\tau_{1}\right) a\left(k+\tau_{1}\right)\left|x_{1}(k)-y_{1}(k)\right| \\
& -\sum_{s=k+1}^{k+\tau_{1}} a(s) M_{1} P_{2}(k) a(k)\left|x_{1}\left(k-\tau_{1}\right)-y_{1}\left(k-\tau_{1}\right)\right| \\
& +\sum_{s=k+\sigma_{1}+1}^{k+\sigma_{1}+\tau_{1}} a(s) M_{1} P_{2}\left(k+\sigma_{1}\right) d\left(k+\sigma_{1}\right)\left|u_{1}(k)-v_{1}(k)\right| \\
& -\sum_{s=k+1}^{k+\tau_{1}} a(s) M_{1} P_{2}(k) d(k)\left|u_{1}\left(k-\sigma_{1}\right)-v_{1}\left(k-\sigma_{1}\right)\right| .
\end{aligned}
$$

We now define

$$
V_{1}(k)=V_{11}(k)+V_{12}(k)+V_{13}(k) .
$$


Then for all $k>k_{0}+\tau$, it follows from (4.14)-(4.24) that

$$
\begin{aligned}
\Delta V_{1} \leq & -\left[\frac{1}{\xi_{1}(k)}-\left|\frac{1}{\xi_{1}(k)}-a(k)\right|\right]\left|x_{1}(k)-y_{1}(k)\right|+d\left(k+\sigma_{1}\right)\left|u_{1}(k)-v_{1}(k)\right| \\
& +E_{1}(k)\left|x_{1}(k)-y_{1}(k)\right|+E_{2}(k)\left|x_{2}(k)-y_{2}(k)\right| \\
& +\sum_{s=k+1}^{k+\tau_{1}} a(s)\left\{\left[P_{1}(k) G_{1}(k)+M_{1} P_{2}(k) E_{1}(k)\right]\left|x_{1}(k)-y_{1}(k)\right|\right. \\
& \left.+M_{1} P_{2}(k) E_{2}(k)\left|x_{2}(k)-y_{2}(k)\right|\right\} \\
& +\sum_{s=k+\tau_{1}+1}^{k+2 \tau_{1}} a(s) M_{1} P_{2}\left(k+\tau_{1}\right) a\left(k+\tau_{1}\right)\left|x_{1}(k)-y_{1}(k)\right| \\
& +\sum_{s=k+\sigma_{1}+1}^{k+\sigma_{1}+\tau_{1}} a(s) M_{1} P_{2}\left(k+\sigma_{1}\right) d\left(k+\sigma_{1}\right)\left|u_{1}(k)-v_{1}(k)\right| .
\end{aligned}
$$

Similarly, we define

$$
V_{2}(k)=V_{21}(k)+V_{22}(k)+V_{23}(k),
$$

where

$$
\begin{gathered}
V_{21}(k)=\left|\ln x_{2}(k)-\ln y_{2}(k)\right|, \\
V_{22}(k)=\sum_{s=k-\tau_{2}}^{k-1} \frac{M_{2}}{m_{1}^{2}} f\left(s+\tau_{2}\right)\left|x_{1}(s)-y_{1}(s)\right|+\sum_{s=k-\sigma_{2}}^{k-1} p\left(s+\sigma_{2}\right)\left|u_{2}(s)-v_{2}(s)\right| \\
+\sum_{s=k}^{k-1+\tau_{2}} \frac{f(s)}{m_{1}} \sum_{u=s-\tau_{2}}^{k-1}\left\{Q_{1}(u) G_{2}(u)\left|x_{2}(u)-y_{2}(u)\right|\right. \\
+M_{2} Q_{2}(u)\left[\frac{f(u)}{m_{1}}\left|x_{2}\left(u-\tau_{2}\right)-y_{2}\left(u-\tau_{2}\right)\right|\right. \\
+\frac{M_{2}}{m_{1}^{2}} f(u)\left|x_{1}\left(u-\tau_{2}\right)-y_{1}\left(u-\tau_{2}\right)\right| \\
\left.\left.+p(u)\left|u_{2}\left(u-\sigma_{2}\right)-v_{2}\left(u-\sigma_{2}\right)\right|\right]\right\},
\end{gathered}
$$


Abstract and Applied Analysis

$$
\begin{aligned}
V_{23}(k)= & \frac{M_{2}}{m_{1}} \sum_{l=k-\tau_{2}}^{k-1} Q_{2}\left(l+\tau_{2}\right) f\left(l+\tau_{2}\right)\left|x_{2}(l)-y_{2}(l)\right| \sum_{s=l+\tau_{2}+1}^{l+2 \tau_{2}} \frac{f(s)}{m_{1}} \\
& +\frac{M_{2}^{2}}{m_{1}^{2}} \sum_{l=k-\tau_{2}}^{k-1} Q_{2}\left(l+\tau_{2}\right) f\left(l+\tau_{2}\right)\left|x_{1}(l)-y_{1}(l)\right| \sum_{s=l+\tau_{2}+1}^{l+2 \tau_{2}} \frac{f(s)}{m_{1}} \\
& +M_{2} \sum_{l=k-\sigma_{2}}^{k-1} Q_{2}\left(l+\sigma_{2}\right) p\left(l+\sigma_{2}\right)\left|u_{2}(l)-v_{2}(l)\right| \sum_{s=l+\sigma_{2}+1}^{l+\sigma_{2}+\tau_{2}} \frac{f(s)}{m_{1}} \\
& G_{2}(s)=g(s)+f(s) \frac{y_{2}\left(s-\tau_{2}\right)}{y_{1}\left(s-\tau_{2}\right)}+p(s) v_{2}\left(s-\sigma_{2}\right) .
\end{aligned}
$$

Thus for all $k>k_{0}+\tau$, it follows from (4.27)-(4.30) that

$$
\begin{aligned}
\Delta V_{2} \leq & -\left[\frac{1}{\xi_{2}(k)}-\left|\frac{1}{\xi_{2}(k)}-\frac{f(k)}{x_{1}\left(k-\tau_{2}\right)}\right|\right]\left|x_{2}(k)-y_{2}(k)\right| \\
& +\frac{M_{2}}{m_{1}^{2}} f\left(k+\tau_{2}\right)\left|x_{1}(k)-y_{1}(k)\right|+p\left(k+\sigma_{2}\right)\left|u_{2}(k)-v_{2}(k)\right| \\
& +\sum_{s=k+1}^{k+\tau_{2}} \frac{f(s)}{m_{1}} Q_{1}(k) G_{2}(k)\left|x_{2}(k)-y_{2}(k)\right| \\
& +\sum_{s=k+\tau_{2}+1}^{k+2 \tau_{2}} \frac{f(s)}{m_{1}} \frac{M_{2}}{m_{1}} Q_{2}\left(k+\tau_{2}\right) f\left(k+\tau_{2}\right)\left|x_{2}(k)-y_{2}(k)\right| \\
& +\sum_{s=k+\tau_{2}+1}^{k+2 \tau_{2}} \frac{f(s)}{m_{1}} \frac{M_{2}^{2}}{m_{1}^{2}} Q_{2}\left(k+\tau_{2}\right) f\left(k+\tau_{2}\right)\left|x_{1}(k)-y_{1}(k)\right| \\
& +\sum_{s=k+\sigma_{2}+1}^{k+\sigma_{2}+\tau_{2}} \frac{f(s)}{m_{1}} M_{2} Q_{2}\left(k+\sigma_{2}\right) p\left(k+\sigma_{2}\right)\left|u_{2}(k)-v_{2}(k)\right| .
\end{aligned}
$$

For $i=1,2$, we define

$$
V_{i}^{\prime}(k)=\left|u_{i}(k)-v_{i}(k)\right|+\sum_{s=k-\rho_{i}}^{k-1} \beta_{i}\left(s+\rho_{i}\right)\left|x_{i}(s)-y_{i}(s)\right|
$$

By calculation, it derives that

$$
\Delta V_{i}^{\prime} \leq-\alpha_{i}(k)\left|u_{i}(k)-v_{i}(k)\right|+\beta_{i}\left(k+\rho_{i}\right)\left|x_{i}(k)-y_{i}(k)\right| .
$$

We now define a Lyapunov function as

$$
V(k)=s_{1} V_{1}(k)+s_{2} V_{2}(k)+\omega_{1} V_{1}^{\prime}(k)+\omega_{2} V_{2}^{\prime}(k) .
$$


It is easy to see that $V(k)>0$ and $V\left(k_{0}+\tau\right)<+\infty$. Calculating the difference of $V$ along the solution of system (1.4), we have that for $k \geq k_{0}+\tau$,

$$
\begin{aligned}
\Delta V \leq & \left\{s_{1}\left[-\min \left(a^{l}, \frac{2}{M_{1}}-a^{u}\right)+E_{1}^{u}+\tau_{1} a^{u}\left[P_{1}^{u} G_{1}^{u}+M_{1} P_{2}^{u} E_{1}^{u}+M_{1} P_{2}^{u} a^{u}\right]\right]\right. \\
& \left.+s_{2} \frac{M_{2}}{m_{1}^{2}} f^{u}\left[1+\tau_{2} \frac{M_{2}}{m_{1}} f^{u} Q_{2}^{u}\right]+\omega_{1} \beta_{1}^{u}\right\}\left|x_{1}(k)-y_{1}(k)\right| \\
& +\left\{s_{2}\left[-\min \left(\frac{f^{l}}{M_{1}}, \frac{2}{M_{2}}-\frac{f^{u}}{m_{1}}\right)+\tau_{2} \frac{f^{u}}{m_{1}}\left[Q_{1}^{u} G_{2}^{u}+\frac{M_{2}}{m_{1}} Q_{2}^{u} f^{u}\right]\right]\right. \\
& \left.+s_{1} E_{2}^{u}\left[1+\tau_{1} a^{u} M_{1} P_{2}^{u}\right]+\omega_{2} \beta_{2}^{u}\right\}\left|x_{2}(k)-y_{2}(k)\right| \\
& +\left\{-\omega_{1} \alpha_{1}^{l}+s_{1} d^{u}\left[1+\tau_{1} a^{u} M_{1} P_{2}^{u}\right]\right\}\left|u_{1}(k)-v_{1}(k)\right| \\
& +\left\{-\omega_{2} \alpha_{2}^{l}+s_{2} p^{u}\left[1+\tau_{2} \frac{f^{u}}{m_{1}} M_{2} Q_{2}^{u}\right]\right\}\left|u_{2}(k)-v_{2}(k)\right| \\
\leq & -\left[s_{1} \mu_{1}-s_{2} \mu_{2}-\omega_{1} \beta_{1}^{u}\right]\left|x_{1}(k)-y_{1}(k)\right|-\left[s_{2} v_{1}-s_{1} v_{2}-\omega_{2} \beta_{2}^{u}\right]\left|x_{2}(k)-y_{2}(k)\right| \\
& -\left[\omega_{1} \alpha_{1}^{l}-s_{1} Q_{1}\right]\left|u_{1}(k)-v_{1}(k)\right|-\left[\omega_{2} \alpha_{2}^{l}-s_{2} \varrho_{2}\right]\left|u_{2}(k)-v_{2}(k)\right| \\
\leq & -\alpha \sum_{i=1}^{2}\left(\left|x_{i}(k)-y_{i}(k)\right|+\left|u_{i}(k)-v_{i}(k)\right|\right),
\end{aligned}
$$

where

$$
\begin{gathered}
\mu_{1}=\min \left(a^{l}, \frac{2}{M_{1}}-a^{u}\right)-E_{1}^{u}-\tau_{1} a^{u}\left[P_{1}^{u} G_{1}^{u}+M_{1} P_{2}^{u} E_{1}^{u}+M_{1} P_{2}^{u} a^{u}\right], \\
\mu_{2}=\frac{M_{2}}{m_{1}^{2}} f^{u}\left[1+\tau_{2} \frac{M_{2}}{m_{1}} Q_{2}^{u} f^{u}\right], \\
v_{1}=\min \left(\frac{f^{l}}{M_{1}}, \frac{2}{M_{2}}-\frac{f^{u}}{m_{1}}\right)-\tau_{2} \frac{f^{u}}{m_{1}}\left[Q_{1}^{u} G_{2}^{u}+\frac{M_{2}}{m_{1}} Q_{2}^{u} f^{u}\right], \\
v_{2}=E_{2}^{u}\left[1+\tau_{1} a^{u} M_{1} P_{2}^{u}\right], \\
\varrho_{1}=d^{u}\left[1+\tau_{1} a^{u} M_{1} P_{2}^{u}\right], \\
Q_{2}=p^{u}\left[1+\tau_{2} \frac{f^{u}}{m_{1}} M_{2} Q_{2}^{u}\right] .
\end{gathered}
$$


Summing both sides of (4.36) from $k_{0}+\tau$ to $k$, it derives that

$$
\sum_{s=k_{0}+\tau}^{k}[V(s+1)-V(s)] \leq-\alpha \sum_{s=k_{0}+\tau}^{k} \sum_{i=1}^{2}\left(\left|x_{i}(s)-y_{i}(s)\right|+\left|u_{i}(s)-v_{i}(s)\right|\right)
$$

It then follows from (4.38) that for $k>k_{0}+\tau$,

$$
V(k+1)+\alpha \sum_{s=k_{0}+\tau}^{k} \sum_{i=1}^{2}\left(\left|x_{i}(s)-y_{i}(s)\right|+\left|u_{i}(s)-v_{i}(s)\right|\right) \leq V\left(k_{0}+\tau\right),
$$

that is

$$
\sum_{s=k_{0}+\tau}^{k} \sum_{i=1}^{2}\left(\left|x_{i}(s)-y_{i}(s)\right|+\left|u_{i}(s)-v_{i}(s)\right|\right) \leq \frac{V\left(k_{0}+\tau\right)}{\alpha}
$$

Then

$$
\sum_{k=k_{0}+\tau}^{\infty} \sum_{i=1}^{2}\left(\left|x_{i}(s)-y_{i}(s)\right|+\left|u_{i}(s)-v_{i}(s)\right|\right) \leq \frac{V\left(k_{0}+\tau\right)}{\alpha}<+\infty
$$

from which we see that

$$
\lim _{k \rightarrow+\infty}\left|x_{i}(k)-y_{i}(k)\right|=0, \quad \lim _{k \rightarrow+\infty}\left|u_{i}(k)-v_{i}(k)\right|=0, \quad i=1,2
$$

This completes the proof of Theorem 4.3.

\section{Almost Periodic Solutions}

In this section, we consider the almost periodic property of system (1.4).

We assume that $a^{*}(k) \in H(a(k)), b^{*}(k) \in H(b(k)), c^{*}(k) \in H(c(k)), d^{*}(k) \in$ $H(d(k)), f^{*}(k) \in H(f(k)), g^{*}(k) \in H(g(k)), p^{*}(k) \in H(p(k)), \alpha_{i}^{*}(k) \in H\left(\alpha_{i}(k)\right), \beta_{i}^{*}(k) \in$ $H\left(\beta_{i}(k)\right), i=1,2$. By Definitions 2.3 and 2.4, there exists an integer valued sequence $\gamma_{n}$ with $\gamma_{n} \rightarrow \infty$ as $n \rightarrow \infty$ such that for $i=1,2, k \in Z$

$$
\begin{array}{lll}
a\left(k+\gamma_{n}\right) \longrightarrow a^{*}(k), & b\left(k+\gamma_{n}\right) \longrightarrow b^{*}(k), & c\left(k+\gamma_{n}\right) \longrightarrow c^{*}(k), \\
d\left(k+\gamma_{n}\right) \longrightarrow d^{*}(k), & f\left(k+\gamma_{n}\right) \longrightarrow f^{*}(k), & g\left(k+\gamma_{n}\right) \longrightarrow g^{*}(k), \\
p\left(k+\gamma_{n}\right) \longrightarrow p^{*}(k), & \alpha_{i}\left(k+\gamma_{n}\right) \longrightarrow \alpha_{i}^{*}(k), & \beta_{i}\left(k+\gamma_{n}\right) \longrightarrow \beta_{i}^{*}(k) .
\end{array}
$$


Then we have the following Hull equation of system (1.4)

$$
\begin{gathered}
x_{1}(k+1)=x_{1}(k) \exp \left[b^{*}(k)-a^{*}(k) x_{1}\left(k-\tau_{1}\right)-\frac{c^{*}(k) x_{1}(k) x_{2}(k)}{h^{2} x_{2}^{2}(k)+x_{1}^{2}(k)}-d^{*}(k) u_{1}\left(k-\sigma_{1}\right)\right], \\
x_{2}(k+1)=x_{2}(k) \exp \left[g^{*}(k)-f^{*}(k) \frac{x_{2}\left(k-\tau_{2}\right)}{x_{1}\left(k-\tau_{2}\right)}-p^{*}(k) u_{2}\left(k-\sigma_{2}\right)\right], \\
\Delta u_{i}(k)=-\alpha_{i}^{*}(k) u_{i}(k)+\beta_{i}^{*}(k) x_{i}\left(k-\rho_{i}\right), \quad i=1,2 .
\end{gathered}
$$

According to the almost periodic theory, we know that if system (1.4) satisfies $\left(H_{1}\right)-\left(H_{3}\right)$, then Hull equation (5.2) also satisfies $\left(H_{1}\right)-\left(H_{3}\right)$.

The following Lemma is Lemma 4.1 in [15].

Lemma 5.1. If each hull equation of system (1.4) has a unique strictly positive solution, then the almost periodic difference system (1.4) has a unique strictly positive almost periodic solution.

Theorem 5.2. Assume that $\left(H_{1}\right)-\left(H_{3}\right)$ holds. Then the almost periodic difference system (1.4) has a unique strictly positive almost periodic solution which is globally attractive.

Proof. We divided the proof into two steps.

Step 1 . We prove the existence of a strictly positive solution of each Hull equation (5.2).

By the almost periodicity of the parameters of system (1.4), there exists an integervalued sequence $\gamma_{n}$ with $\gamma_{n} \rightarrow \infty$ as $n \rightarrow \infty$ such that for $i=1,2, k \in Z$

$$
\begin{array}{lll}
a^{*}\left(k+\gamma_{n}\right) \longrightarrow a^{*}(k), & b^{*}\left(k+\gamma_{n}\right) \longrightarrow b^{*}(k), & c^{*}\left(k+\gamma_{n}\right) \longrightarrow c^{*}(k), \\
d^{*}\left(k+\gamma_{n}\right) \longrightarrow d^{*}(k), & f^{*}\left(k+\gamma_{n}\right) \longrightarrow f^{*}(k), & g^{*}\left(k+\gamma_{n}\right) \longrightarrow g^{*}(k), \\
p^{*}\left(k+\gamma_{n}\right) \longrightarrow p^{*}(k), & \alpha_{i}^{*}\left(k+\gamma_{n}\right) \longrightarrow \alpha_{i}^{*}(k), & \beta_{i}^{*}\left(k+\gamma_{n}\right) \longrightarrow \beta_{i}^{*}(k) .
\end{array}
$$

Suppose that $X(k)=\left(x_{1}(k), x_{2}(k), u_{1}(k), u_{2}(k)\right)^{T}$ is any positive solution of Hull equation (5.2). Since $\left(H_{1}\right),\left(H_{2}\right)$ hold, according to Theorem 3.1, we obtain

$$
\begin{gathered}
m_{i} \leq \liminf _{k \rightarrow+\infty} x_{i}(k) \leq \limsup _{k \rightarrow+\infty} x_{i}(k) \leq M_{i} \\
n_{i} \leq \liminf _{k \rightarrow+\infty} u_{i}(k) \leq \limsup _{k \rightarrow+\infty} u_{i}(k) \leq N_{i}, \quad i=1,2 .
\end{gathered}
$$

Therefore, one has

$$
\begin{gathered}
0<\inf _{k \in Z^{+}} x_{i}(k) \leq \sup _{k \in Z^{+}} x_{i}(k)<\infty, \\
0<\inf _{k \in Z^{+}} u_{i}(k) \leq \sup _{k \in Z^{+}} u_{i}(k)<\infty, \quad i=1,2 .
\end{gathered}
$$


For any $\varepsilon>0$ small enough, it follows from Theorem 3.1 that there exists enough large $K_{0}>K_{5}+\tau$ such that for $i=1,2$ and $k \geq K_{0}$

$$
m_{i}-\varepsilon \leq x_{i}(k) \leq M_{i}+\varepsilon, \quad n_{i}-\varepsilon \leq u_{i}(k) \leq N_{i}+\varepsilon
$$

Let $x_{n}(k)=x_{n}\left(k+\gamma_{n}\right)$ and $u_{n}(k)=u_{n}\left(k+\gamma_{n}\right)$ for $k \geq K_{0}+\tau-\gamma_{n}, n=1,2, \ldots$. For any positive integer $q$ and $i=1,2$, it is not difficult to show that there are sequences $\left\{x_{i n}(k)\right.$ : $n \geq q\}$ and $\left\{u_{i n}(k): n \geq q\right\}$ such that the sequences $\left\{x_{i n}(k)\right\}$ and $\left\{u_{i n}(k)\right\}$ have subsequences, denoted by $\left\{x_{i n}(k)\right\}$ and $\left\{u_{i n}(k)\right\}$ again, converging on any finite interval of $Z$ as $n \rightarrow \infty$, respectively. So we have sequences $\left\{y_{i}(k)\right\}$ and $\left\{v_{i}(k)\right\} i=1,2$ such that for $k \in Z, n \rightarrow \infty$

$$
\begin{aligned}
& x_{\text {in }}(k) \longrightarrow y_{i}(k), \quad u_{\text {in }}(k) \longrightarrow v_{i}(k), \\
& x_{1}(k+1)=x_{1}(k) \exp \left[b^{*}\left(k+\gamma_{n}\right)-a^{*}\left(k+\gamma_{n}\right) x_{1}\left(k-\tau_{1}\right)\right. \\
& \left.-\frac{c^{*}\left(k+\gamma_{n}\right) x_{1}(k) x_{2}(k)}{h^{2} x_{2}^{2}(k)+x_{1}^{2}(k)}-d^{*}\left(k+\gamma_{n}\right) u_{1}\left(k-\sigma_{1}\right)\right] \\
& x_{2}(k+1)=x_{2}(k) \exp \left[g^{*}\left(k+\gamma_{n}\right)-f^{*}\left(k+\gamma_{n}\right) \frac{x_{2}\left(k-\tau_{2}\right)}{x_{1}\left(k-\tau_{2}\right)}-p^{*}\left(k+\gamma_{n}\right) u_{2}\left(k-\sigma_{2}\right)\right] \text {, } \\
& \Delta u_{i}(k)=-\alpha_{i}^{*}\left(k+\gamma_{n}\right) u_{i}(k)+\beta_{i}^{*}\left(k+\gamma_{n}\right) x_{i}\left(k-\rho_{i}\right), \quad i=1,2,
\end{aligned}
$$

which implies

$$
\begin{gathered}
y_{1}(k+1)=y_{1}(k) \exp \left[b^{*}(k)-a^{*}(k) y_{1}\left(k-\tau_{1}\right)-\frac{c^{*}(k) y_{1}(k) y_{2}(k)}{h^{2} y_{2}^{2}(k)+y_{1}^{2}(k)}-d^{*}(k) v_{1}\left(k-\sigma_{1}\right)\right], \\
y_{2}(k+1)=y_{2}(k) \exp \left[g^{*}(k)-f^{*}(k) \frac{y_{2}\left(k-\tau_{2}\right)}{y_{1}\left(k-\tau_{2}\right)}-p^{*}(k) v_{2}\left(k-\sigma_{2}\right)\right], \\
\Delta v_{i}(k)=-\alpha_{i}^{*}(k) v_{i}(k)+\beta_{i}^{*}(k) y_{i}\left(k-\rho_{i}\right), \quad i=1,2 .
\end{gathered}
$$

Thus we can easily see that $Y(k)=\left(y_{1}(k), y_{2}(k), v_{1}(k), v_{2}(k)\right)^{T}$ is a solution of Hull equation (5.2) and $m_{i}-\varepsilon \leq y_{i}(k) \leq M_{i}+\varepsilon, n_{i}-\varepsilon \leq v_{i}(k) \leq N_{i}+\varepsilon$, for $i=1,2, k \in Z$. Since $\varepsilon$ is an arbitrary small positive number, it follows that

$$
\begin{gathered}
0<\inf _{k \in Z^{+}} y_{i}(k) \leq \sup _{k \in Z^{+}} y_{i}(k)<\infty, \\
0<\inf _{k \in Z^{+}} v_{i}(k) \leq \sup _{k \in Z^{+}} v_{i}(k)<\infty, \quad i=1,2 .
\end{gathered}
$$

This completes the proof of Step 1. (5.2).

Step 2. We show the uniqueness of the strictly positive solution of each Hull equation 
Suppose that the Hull equation (5.2) has two arbitrary strictly positive solutions $X^{*}(k)=\left(x_{1}^{*}(k), x_{2}^{*}(k), u_{1}^{*}(k), u_{2}^{*}(k)\right)^{T}$ and $Y^{*}(k)=\left(y_{1}^{*}(k), y_{2}^{*}(k), v_{1}^{*}(k), v_{2}^{*}(k)\right)^{T}$. Now we define a Lyapunov function $V^{*}(k)$ on $k \in Z$ as follows:

$$
V^{*}(k)=\sum_{i=1}^{2} s_{i}\left(V_{i 1}^{*}(k)+V_{i 2}^{*}(k)+V_{i 3}^{*}(k)\right)+\sum_{i=1}^{2} \omega_{i} V_{i}^{\prime *}(k)
$$

where

$$
\begin{aligned}
& V_{i 1}^{*}(k)=\left|\ln x_{i}^{*}(k)-\ln y_{i}^{*}(k)\right|, \quad i=1,2, \\
& V_{12}^{*}(k)=\sum_{s=k-\sigma_{1}}^{k-1} d\left(s+\sigma_{1}\right)\left|u_{1}^{*}(s)-v_{1}^{*}(s)\right| \\
& +\sum_{s=k}^{k-1+\tau_{1}} a(s) \sum_{u=s-\tau_{1}}^{k-1}\left\{\left[P_{1}(u) G_{1}(u) M_{1} P_{2}(u) E_{1}(u)\right]\left|x_{1}^{*}(u)-y_{1}^{*}(u)\right|\right. \\
& +M_{1} P_{2}(u)\left[a(u)\left|x_{1}^{*}\left(u-\tau_{1}\right)-y_{1}^{*}\left(u-\tau_{1}\right)\right|\right. \\
& +d(u)\left|u_{1}^{*}\left(u-\sigma_{1}\right)-v_{1}^{*}\left(u-\sigma_{1}\right)\right| \\
& \left.\left.+E_{2}(u)\left|x_{2}^{*}(u)-y_{2}^{*}(u)\right|\right]\right\}, \\
& V_{13}^{*}(k)=M_{1} \sum_{l=k-\tau_{1}}^{k-1} P_{2}\left(l+\tau_{1}\right) a\left(l+\tau_{1}\right)\left|x_{1}^{*}(l)-y_{1}^{*}(l)\right| \sum_{s=l+\tau_{1}+1}^{l+2 \tau_{1}} a(s) \\
& +M_{1} \sum_{l=k-\sigma_{1}}^{k-1} P_{2}\left(l+\sigma_{1}\right) d\left(l+\sigma_{1}\right)\left|u_{1}^{*}(l)-v_{1}^{*}(l)\right| \sum_{s=l+\sigma_{1}+1}^{l+\sigma_{1}+\tau_{1}} a(s), \\
& V_{22}^{*}(k)=\sum_{s=k-\tau_{2}}^{k-1} \frac{M_{2}}{m_{1}^{2}} f\left(s+\tau_{2}\right)\left|x_{1}^{*}(s)-y_{1}^{*}(s)\right|+\sum_{s=k-\sigma_{2}}^{k-1} p\left(s+\sigma_{2}\right)\left|u_{2}^{*}(s)-v_{2}^{*}(s)\right| \\
& +\sum_{s=k}^{k-1+\tau_{2}} \frac{f(s)}{m_{1}} \sum_{u=s-\tau_{2}}^{k-1}\left\{Q_{1}(u) G_{2}(u)\left|x_{2}^{*}(u)-y_{2}^{*}(u)\right|\right. \\
& +M_{2} Q_{2}(u)\left[\frac{f(u)}{m_{1}}\left|x_{2}^{*}\left(u-\tau_{2}\right)-y_{2}^{*}\left(u-\tau_{2}\right)\right|\right. \\
& +\frac{M_{2}}{m_{1}^{2}} f(u)\left|x_{1}^{*}\left(u-\tau_{2}\right)-y_{1}^{*}\left(u-\tau_{2}\right)\right| \\
& \left.\left.+p(u)\left|u_{2}^{*}\left(u-\sigma_{2}\right)-v_{2}^{*}\left(u-\sigma_{2}\right)\right|\right]\right\},
\end{aligned}
$$


Abstract and Applied Analysis

$$
\begin{aligned}
V_{23}^{*}(k)= & \frac{M_{2}}{m_{1}} \sum_{l=k-\tau_{2}}^{k-1} Q_{2}\left(l+\tau_{2}\right) f\left(l+\tau_{2}\right)\left|x_{2}^{*}(l)-y_{2}^{*}(l)\right| \sum_{s=l+\tau_{2}+1}^{l+2 \tau_{2}} \frac{f(s)}{m_{1}} \\
& +\frac{M_{2}^{2}}{m_{1}^{2}} \sum_{l=k-\tau_{2}}^{k-1} Q_{2}\left(l+\tau_{2}\right) f\left(l+\tau_{2}\right)\left|x_{1}^{*}(l)-y_{1}^{*}(l)\right| \sum_{s=l+\tau_{2}+1}^{l+2 \tau_{2}} \frac{f(s)}{m_{1}} \\
& +M_{2} \sum_{l=k-\sigma_{2}}^{k-1} Q_{2}\left(l+\sigma_{2}\right) p\left(l+\sigma_{2}\right)\left|u_{2}^{*}(l)-v_{2}^{*}(l)\right| \sum_{s=l+\sigma_{2}+1}^{l+\sigma_{2}+\tau_{2}} \frac{f(s)}{m_{1}} \\
V_{i}^{*}(k) & =\left|u_{i}^{*}(k)-v_{i}^{*}(k)\right|+\sum_{s=k-\rho_{i}}^{k-1} \beta_{i}\left(s+\rho_{i}\right)\left|x_{i}^{*}(s)-y_{i}^{*}(s)\right|
\end{aligned}
$$

where $E_{i}, G_{i}, P_{i}, Q_{i}, i=1,2$ are defined by Theorem 4.3 .

Similar to the discussion of (3.32), calculating the difference of $V^{*}(k)$ along the solution of the Hull equation (5.2), one has

$$
\Delta V^{*} \leq-\alpha \sum_{i=1}^{2}\left(\left|x_{i}^{*}(k)-y_{i}^{*}(k)\right|+\left|u_{i}^{*}(k)-v_{i}^{*}(k)\right|\right), \quad \text { for } k \in Z .
$$

It follows from (5.12) we know that $V^{*}(k)$ is a nonincreasing function on Z. Summing both sides of the above inequalities from $k$ to 0 , we get

$$
\alpha \sum_{s=k}^{0} \sum_{i=1}^{2}\left(\left|x_{i}^{*}(s)-y_{i}^{*}(s)\right|+\left|u_{i}^{*}(s)-v_{i}^{*}(s)\right|\right) \leq V^{*}(k)-V^{*}(0), \quad \text { for } k<0
$$

So we have $\sum_{s=k}^{0} \sum_{i=1}^{2}\left(\left|x_{i}^{*}(s)-y_{i}^{*}(s)\right|+\left|u_{i}^{*}(s)-v_{i}^{*}(s)\right|\right)<+\infty, k \rightarrow-\infty$, thus we obtain

$$
\lim _{k \rightarrow-\infty}\left|x_{i}^{*}(k)-y_{i}^{*}(k)\right|=0, \quad \lim _{k \rightarrow-\infty}\left|u_{i}^{*}(k)-v_{i}^{*}(k)\right|=0, \quad i=1,2 .
$$

From (5.14), for a positive integer $K$, we have

$$
\left|x_{i}^{*}(k)-y_{i}^{*}(k)\right|<\varepsilon, \quad\left|u_{i}^{*}(k)-v_{i}^{*}(k)\right|<\varepsilon, \quad \text { for } k<-K, i=1,2 .
$$


It follows from (5.10) that

$$
\begin{aligned}
& V_{i 1}^{*}(k) \leq \frac{1}{m_{i}}\left|x_{i}^{*}(k)-y_{i}^{*}(k)\right| \leq \frac{1}{m_{i}} \varepsilon, \\
& V_{12}^{*}(k)=\sigma_{1} d^{u} \max _{s \leq k}\left|u_{1}^{*}(s)-v_{1}^{*}(s)\right| \\
& +\left(\tau_{1}\right)^{2} a^{u}\left\{\left[P_{1}^{u} G_{1}^{u}+M_{1} P_{2}^{u} E_{1}^{u}\right] \max _{s \leq k}\left|x_{1}^{*}(s)-y_{1}^{*}(s)\right|\right. \\
& +M_{1} P_{2}^{u}\left[a^{u} \max _{s \leq k}\left|x_{1}^{*}(s)-y_{1}^{*}(s)\right|+d^{u} \max _{s \leq k}\left|u_{1}^{*}(s)-v_{1}^{*}(s)\right|\right. \\
& \left.\left.+E_{2}^{u} \max _{s \leq k}\left|x_{2}^{*}(s)-y_{2}^{*}(s)\right|\right]\right\} \\
& \leq\left\{\sigma_{1} d^{u}+\left(\tau_{1}\right)^{2} a^{u}\left[\left(P_{1}^{u} G_{1}^{u}+M_{1} P_{2}^{u} E_{1}^{u}\right)+M_{1} P_{2}^{u}\left(a^{u}+d^{u}+E_{2}^{u}\right)\right]\right\} \varepsilon^{\prime} \\
& V_{13}^{*}(k) \leq \tau_{1} M_{1} P_{2}^{u} a^{u}\left(\tau_{1} a^{u} \max _{s \leq k}\left|x_{1}^{*}(s)-y_{1}^{*}(s)\right|+\sigma_{1} d^{u} \max _{s \leq k}\left|u_{1}^{*}(s)-v_{1}^{*}(s)\right|\right) \\
& \leq \tau_{1} M_{1} P_{2}^{u} a^{u}\left(\tau_{1} a^{u}+\sigma_{1} d^{u}\right) \varepsilon, \\
& V_{22}^{*}(k) \leq \tau_{2} \frac{M_{2}}{m_{1}^{2}} f^{u} \max _{s \leq k}\left|x_{1}^{*}(s)-y_{1}^{*}(s)\right|+\sigma_{2} p^{u} \max _{s \leq k}\left|u_{2}^{*}(s)-v_{2}^{*}(s)\right| \\
& +\left(\tau_{2}\right)^{2} \frac{f^{u}}{m_{1}}\left\{Q_{1}^{u} G_{2}^{u} \max _{s \leq k}\left|x_{2}^{*}(s)-y_{2}^{*}(s)\right|\right. \\
& +M_{2} Q_{2}^{u}\left[\frac{f^{u}}{m_{1}} \max _{s \leq k}\left|x_{2}^{*}(s)-y_{2}^{*}(s)\right|+\frac{M_{2}}{m_{1}^{2}} f^{u} \max _{s \leq k}\left|x_{1}^{*}(s)-y_{1}^{*}(s)\right|\right. \\
& \left.\left.+p^{u} \max _{s \leq k}\left|u_{2}^{*}(s)-v_{2}^{*}(s)\right|\right]\right\}, \\
& \leq\left\{\tau_{2} \frac{M_{2}}{m_{1}^{2}} f^{u}+\sigma_{2} p^{u}+\left(\tau_{2}\right)^{2} \frac{f^{u}}{m_{1}}\left[Q_{1}^{u} F_{2}^{u}+M_{2} Q_{2}^{u}\left(\frac{f^{u}}{m_{1}}+\frac{M_{2}}{m_{1}^{2}} f^{u}+p^{u}\right)\right]\right\}, \\
& V_{23}^{*}(k) \leq \tau_{2} \frac{f^{u}}{m_{1}} M_{2} Q_{2}^{u}\left(\tau_{2} \frac{f^{u}}{m_{1}} \max _{s \leq k}\left|x_{2}^{*}(s)-y_{2}^{*}(s)\right|+\tau_{2} \frac{M_{2}}{m_{1}^{2}} f^{u} \max _{s \leq k}\left|x_{1}^{*}(s)-y_{1}^{*}(s)\right|\right. \\
& \left.+\sigma_{2} p^{u} \max _{s \leq k}\left|u_{2}^{*}(s)-v_{2}^{*}(s)\right|\right) \\
& \leq \tau_{2} \frac{f^{u}}{m_{1}} M_{2} Q_{2}^{u}\left(\tau_{2} \frac{f^{u}}{m_{1}}+\tau_{2} \frac{M_{2}}{m_{1}^{2}} f^{u}+\sigma_{2} p^{u}\right) \varepsilon, \\
& V_{i}^{\prime *}(k) \leq \max _{s \leq k}\left|u_{i}^{*}(s)-v_{i}^{*}(s)\right|+\rho_{i} \beta_{i}^{u} \max _{s \leq k}\left|x_{i}^{*}(s)-y_{i}^{*}(s)\right| \leq\left(1+\rho_{i} \beta_{i}^{u}\right) \varepsilon .
\end{aligned}
$$


Let

$$
\begin{aligned}
& Q=s_{1}\left\{\frac{1}{m_{1}}+\sigma_{1} d^{u}+\left(\tau_{1}\right)^{2} a^{u}\left[\left(P_{1}^{u} G_{1}^{u}+M_{1} P_{2}^{u} E_{1}^{u}\right)+M_{1} P_{2}^{u}\left(a^{u}+d^{u}+E_{2}^{u}\right)\right]\right. \\
& \left.+\tau_{1} M_{1} P_{2}^{u} a^{u}\left(\tau_{1} a^{u}+\sigma_{1} d^{u}\right)\right\} \\
& +s_{2}\left\{\frac{1}{m_{2}}+\tau_{2} \frac{M_{2}}{m_{1}^{2}} f^{u}+\sigma_{2} p^{u}+\left(\tau_{2}\right)^{2} \frac{f^{u}}{m_{1}}\left[Q_{1}^{u} G_{2}^{u}+M_{2} Q_{2}^{u}\left(\frac{f^{u}}{m_{1}}+\frac{M_{2}}{m_{1}^{2}} f^{u}+p^{u}\right)\right]\right. \\
& \left.+\tau_{2} \frac{f^{u}}{m_{1}} M_{2} Q_{2}^{u}\left(\tau_{2} \frac{f^{u}}{m_{1}}+\tau_{2} \frac{M_{2}}{m_{1}^{2}} f^{u}+\sigma_{2} p^{u}\right)\right\}+\sum_{i=1}^{2} \omega_{i}\left(1+\rho_{i} \beta_{i}^{u}\right) .
\end{aligned}
$$

It follows from (5.10) and the above inequalities that $V^{*}(k) \leq Q \varepsilon$ for $k<-K$, hence $\lim _{k \rightarrow-\infty} V^{*}(k)=0$. Furthermore, $V^{*}(k)$ is a nonincreasing function on $Z$, thus $V^{*}(k) \equiv 0$, that is, $x_{i}^{*}(k)=y_{i}^{*}(k), u_{i}^{*}(k)=v_{i}^{*}(k), i=1,2$ for all $k \in Z$. This ends the proof of Step 2 .

By the above discussion and Theorem 4.3, we conclude that any hull equation of system (1.4) has a unique strictly positive solution. Then the almost periodic system (1.4) has a unique strictly positive almost periodic solution which is globally attractive. The proof of Theorem 5.2 is completed.

\section{Concluding Remarks}

In this paper, a discrete almost periodic ratio-dependent Leslie system with time delays and feedback controls is considered. By applying the difference inequality, some sufficient conditions are established, which are independent of feedback control variables, to ensure the permanence of system (1.4). By constructing the suitable Lyapunov function, we show that system (1.4) is globally attractive under some appropriate conditions. Furthermore, by using an almost periodic functional hull theory, we show that the almost periodic system (1.4) has a unique strictly positive almost periodic solution which is globally attractive.

We would like to mention here the question of whether the feedback control variables have the influence on the stability property of the system or not. It is, in fact, a very challenging problem, and we leave it for our future work.

\section{Acknowledgments}

The authors are grateful to the editor, Wolfgang Ruess, and referees for a number of helpful suggestions that have greatly improved our original submission. This work is supported by the National Natural Science Foundation of China (nos. 71171035, 71173029), China Postdoctoral Science Foundation (no. 2012M511598), and the Teaching and Research Foundation of Dongbei University of Finance and Economics (nos. YY11001, GW11003). 


\section{References}

[1] A. A. Berryman, "The origins and evolution of predator-prey theory," Ecology, vol. 73, no. 5, pp. 15301535, 1992.

[2] J. D. Murray, Mathematical Biology, Springer-Verlag, Berlin, Germany, 1989.

[3] R. M. May, Stability and Complexity in Model Ecosystems, Princeton University Press, Princeton, NJ, USA, 1974.

[4] X. Z. Meng and L. S. Chen, "Almost periodic solution of non-autonomous Lotka-Volterra predatorprey dispersal system with delays," Journal of Theoretical Biology, vol. 243, no. 4, pp. 562-574, 2006.

[5] P. H. Leslie, "Some further notes on the use of matrices in population mathematics," Biometrika, vol. 35, pp. 213-245, 1948.

[6] M. A. Aziz-Alaoui and M. Daher Okiye, "Boundedness and global stability for a predator-prey model with modified Leslie-Gower and Holling-type II schemes," Applied Mathematics Letters, vol. 16, no. 7, pp. 1069-1075, 2003.

[7] X. H. Ding, C. Lu, and M. Liu, "Periodic solutions for a semi-ratio-dependent predator-prey system with nonmonotonic functional response and time delay," Nonlinear Analysis. Real World Applications, vol. 9, no. 3, pp. 762-775, 2008.

[8] H. Y. Lu and W. G. Wang, "Dynamics of a delayed discrete semi-ratio-dependent predator-prey system with Holling type IV functional response," Advances in Difference Equations, vol. 2011, no. 1, p. 7, 2011.

[9] H. Lu and W. Wang, "Dynamics of a nonautonomous Leslie-Gower type food chain model with delays," Discrete Dynamics in Nature and Society, vol. 2011, Article ID 380279, 19 pages, 2011.

[10] A. P. Gutierrez, "Physiological basis of ratio-dependent predator-prey theory: the metabolic pool model as a paradigm," Ecology, vol. 73, no. 5, pp. 1552-1563, 1992.

[11] R. Arditi and H. Saiah, "Empirical evidence of the role of heterogeneity in ratio-dependent consumption," Ecology, vol. 73, no. 5, pp. 1544-1551, 1992.

[12] R. P. Agarwal and P. J. Y. Wong, Advanced Topics in Difference Equations, Kluwer Academic Publishers Group, Dordrecht, The Netherlands, 1997.

[13] F. Chen, L. Wu, and Z. Li, "Permanence and global attractivity of the discrete Gilpin-Ayala type population model," Computers \& Mathematics with Applications, vol. 53, no. 8, pp. 1214-1227, 2007.

[14] F. D. Chen, "Permanence of a discrete N-species food-chain system with time delays," Applied Mathematics and Computation, vol. 185, no. 1, pp. 719-726, 2007.

[15] Z. Li, F. Chen, and M. He, "Almost periodic solutions of a discrete Lotka-Volterra competition system with delays," Nonlinear Analysis. Real World Applications, vol. 12, no. 4, pp. 2344-2355, 2011.

[16] A. M. Fink and G. Seifert, "Liapunov functions and almost periodic solutions for almost periodic systems," Journal of Differential Equations, vol. 5, pp. 307-313, 1969.

[17] S. N. Zhang, "Existence of almost periodic solution for difference systems," Annals Differential Equations, vol. 16, no. 2, pp. 184-206, 2000.

[18] X. H. Ding and C. Lu, "Existence of positive periodic solution for ratio-dependent $N$-species difference system," Applied Mathematical Modelling, vol. 33, no. 6, pp. 2748-2756, 2009.

[19] H. Y. Lu, "Permanence of a discrete nonlinear prey-competition system with delays," Discrete Dynamics in Nature and Society, vol. 2009, Article ID 605254, 15 pages, 2009.

[20] X. T. Yang, "Uniform persistence and periodic solutions for a discrete predator-prey system with delays," Journal of Mathematical Analysis and Applications, vol. 316, no. 1, pp. 161-177, 2006.

[21] F. D. Chen, "Permanence of a discrete $N$-species food-chain system with time delays," Applied Mathematics and Computation, vol. 185, no. 1, pp. 719-726, 2007.

[22] Y.-H. Fan and L.-L. Wang, "Permanence for a discrete model with feedback control and delay," Discrete Dynamics in Nature and Society, vol. 2008, Article ID 945109, 8 pages, 2008.

[23] F. Chen, "Permanence of a discrete $N$-species cooperation system with time delays and feedback controls," Applied Mathematics and Computation, vol. 186, no. 1, pp. 23-29, 2007.

[24] J. Diblík, M. Ružičková, Z. Šmarda, and Z. Šutá, "Asymptotic convergence of the solutions of a dynamic equation on discrete time scales," Abstract and Applied Analysis, vol. 2012, Article ID 580750, 20 pages, 2012.

[25] L. Berezansky, J. Diblík, M. Ružičková, and Z. Šutá, "Asymptotic convergence of the solutions of a discrete equation with two delays in the critical case," Abstract and Applied Analysis, vol. 2011, Article ID 709427, 15 pages, 2011. 
[26] J. Diblík and E. Schmeidel, "On the existence of solutions of linear Volterra difference equations asymptotically equivalent to a given sequence," Applied Mathematics and Computation, vol. 218, no. 18, pp. 9310-9320, 2012.

[27] M. De la Sen, "Stability of impulsive time-varying systems and compactness of the operators mapping the input space into the state and output spaces," Journal of Mathematical Analysis and Applications, vol. 321 , no. 2, pp. 621-650, 2006. 


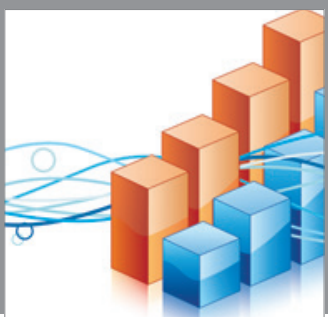

Advances in

Operations Research

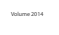

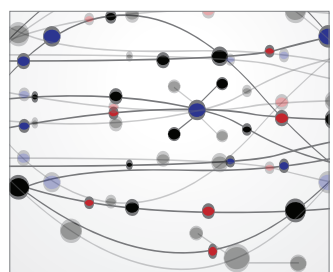

\section{The Scientific} World Journal
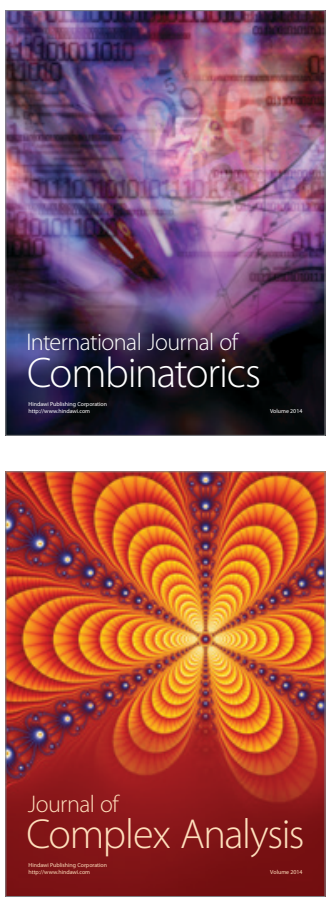

International Journal of

Mathematics and

Mathematical

Sciences
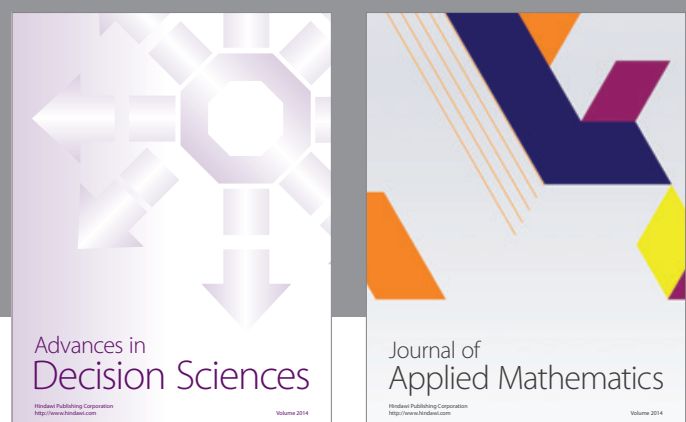

Journal of

Applied Mathematics
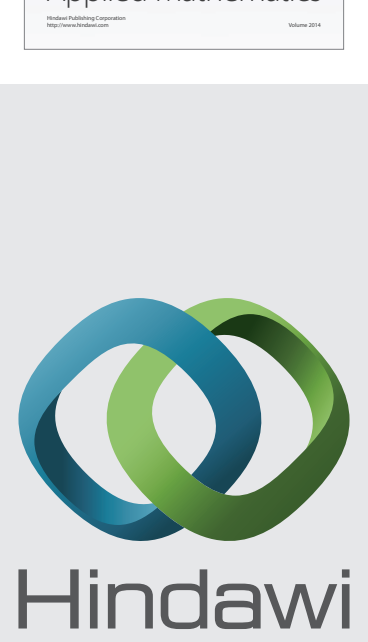

Submit your manuscripts at http://www.hindawi.com
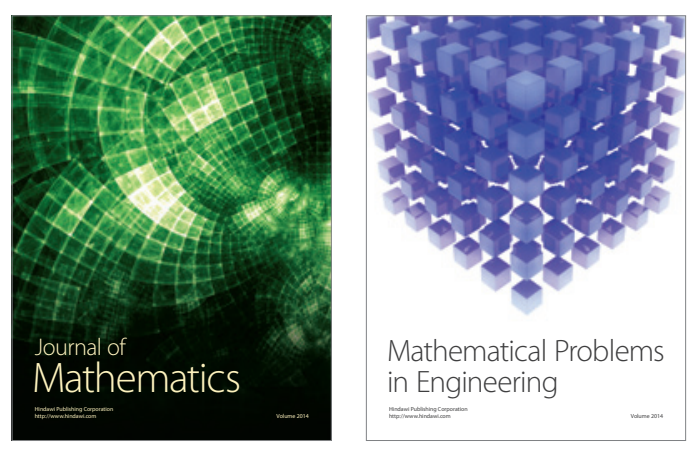

Mathematical Problems in Engineering
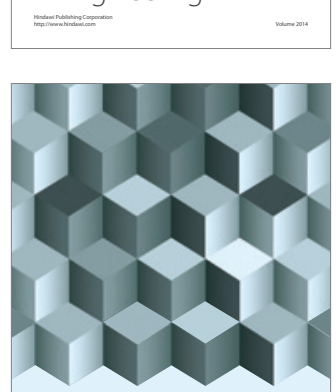

Journal of

Function Spaces
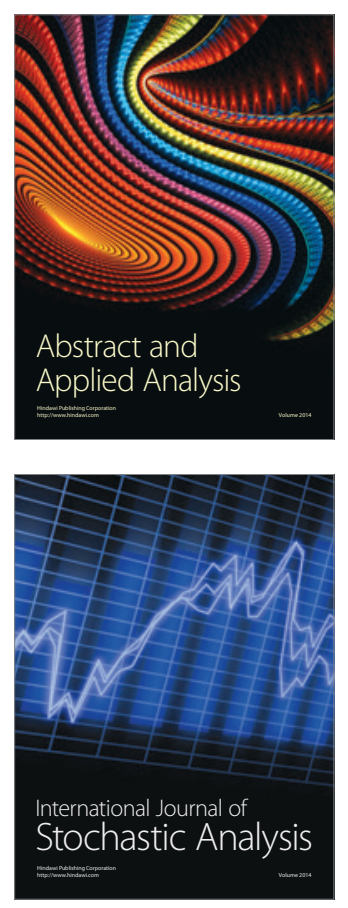

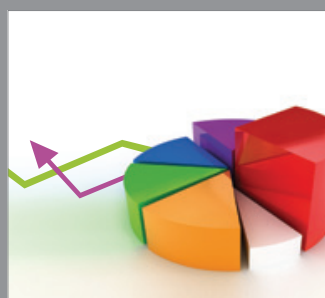

ournal of

Probability and Statistics

Promensencen
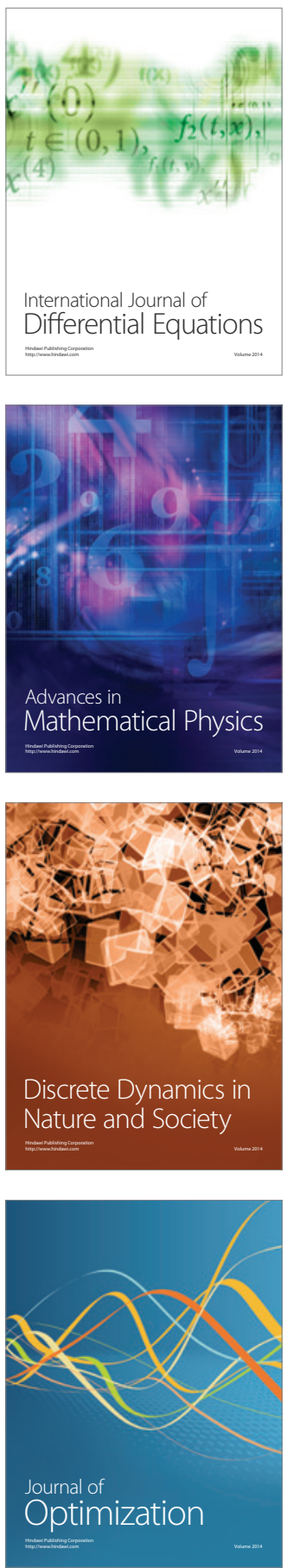